\title{
Ligand-Promoted Iridium-Catalyzed Transfer Hydrogenation of Terminal Alkynes with Ethanol and its Application
}

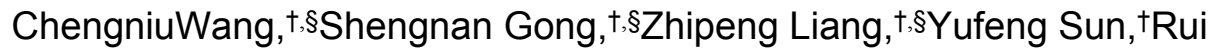

Cheng, ${ }^{\dagger}$ Banghua Yang, ${ }^{\dagger}{ }^{\top}$ irong Liu, ${ }^{\dagger}$ JinfeiYang, ${ }^{\dagger}, *$ and Fei Sun ${ }^{\dagger}, *$

${ }^{\dagger}$ Medical school, Institute of Reproductive Medicine, Nantong University, Nantong 226019

(China)

$\S$ These authors contributed equally to this work.

*jfyang@ntu.edu.cn, sunfei@ntu.edu.cn 


\section{NMR Spectra}

Figure S1. ${ }^{1} \mathrm{H}$ NMR spectrum (400 MHz, $\mathrm{CDCl}_{3}$, TMS) of compound 2a
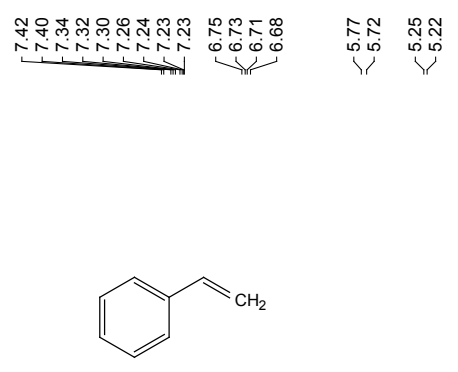

$2 a$

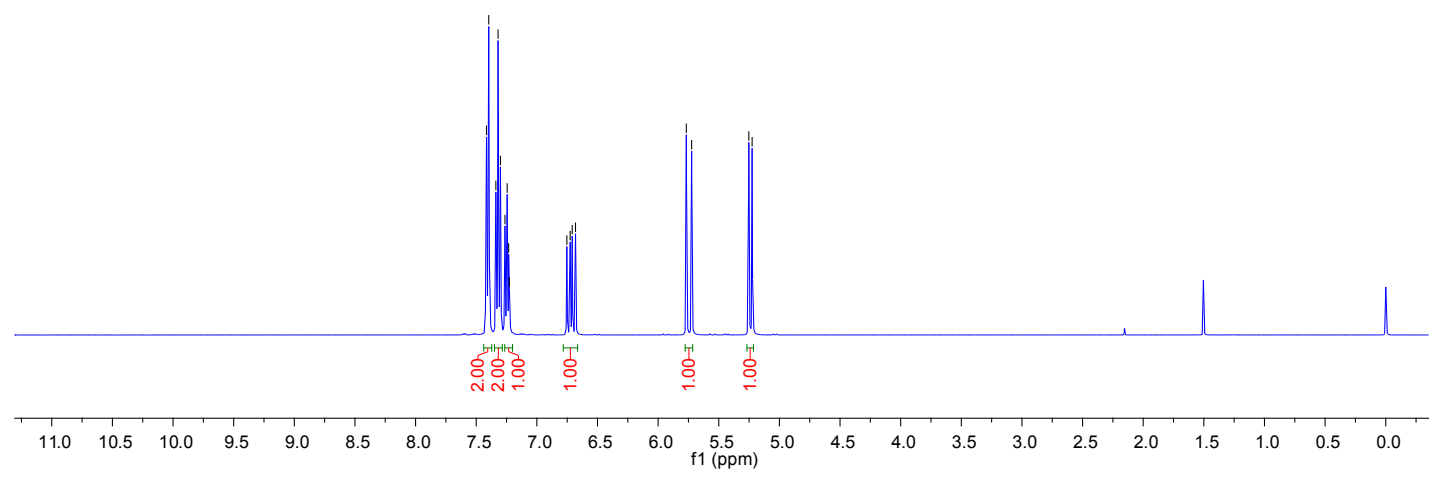

Figure S2. ${ }^{13} \mathrm{C}$ NMR spectrum $\left(100 \mathrm{MHz}, \mathrm{CDCl}_{3}\right)$ of compound 2a
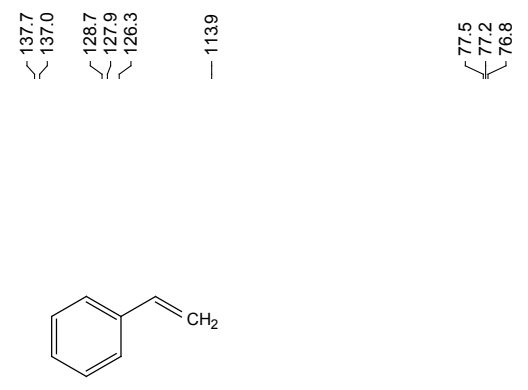

2a

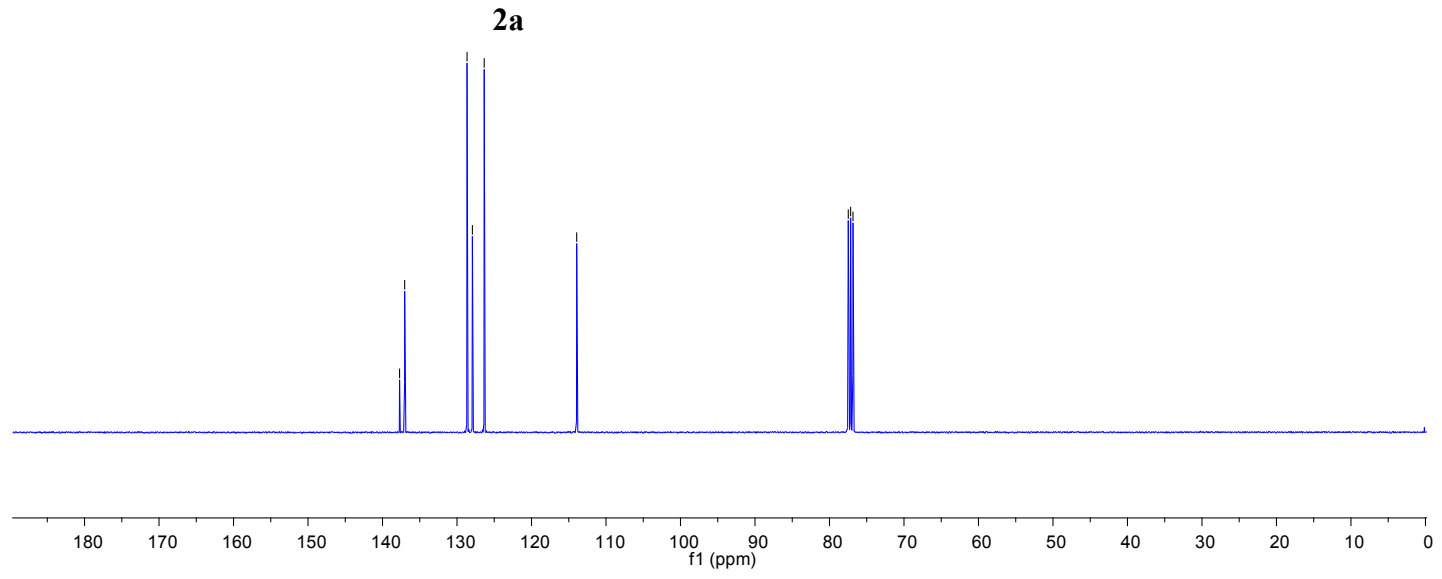


Figure S3. ${ }^{1} \mathrm{H}$ NMR spectrum (400 $\mathrm{MHz}^{-\mathrm{CDCl}_{3}}$, TMS) of compound 2b

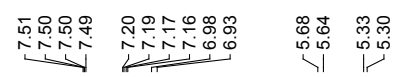

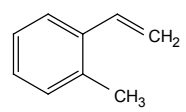

2b

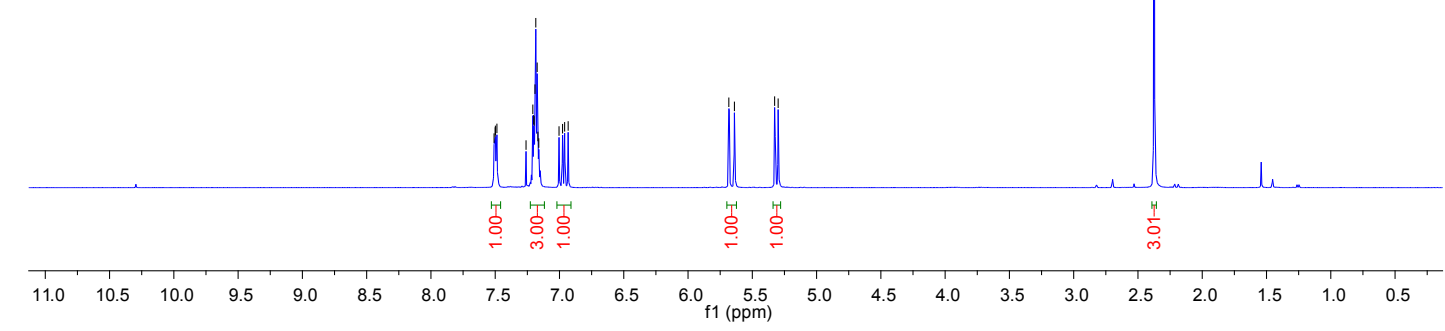

Figure S4. ${ }^{13} \mathrm{C}$ NMR spectrum $\left(100 \mathrm{MHz}, \mathrm{CDCl}_{3}\right)$ of compound $2 \mathrm{~b}$

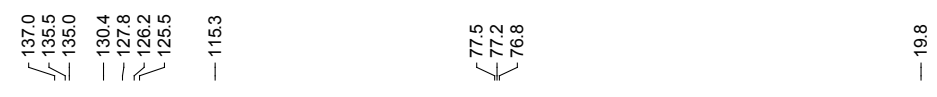

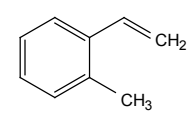

2b

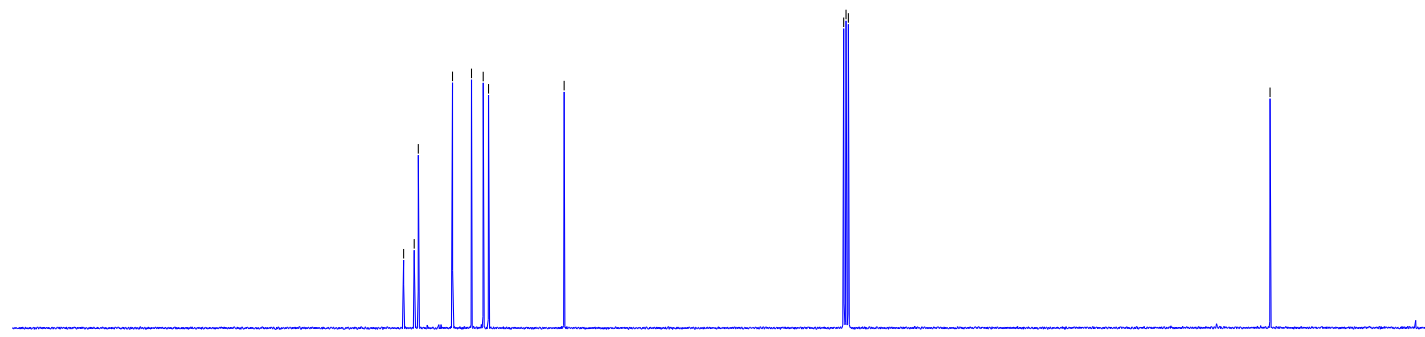

$\begin{array}{llllllllllllllllllll}180 & 170 & 160 & 150 & 140 & 130 & 120 & 110 & 100 & 90 & 80 & 70 & 60 & 50 & 40 & 30 & 20 & 10 & 0\end{array}$ 
Figure S5. ${ }^{1} \mathrm{H}$ NMR spectrum (400 $\mathrm{MHz}^{-\mathrm{CDCl}_{3}}$, TMS) of compound 2c
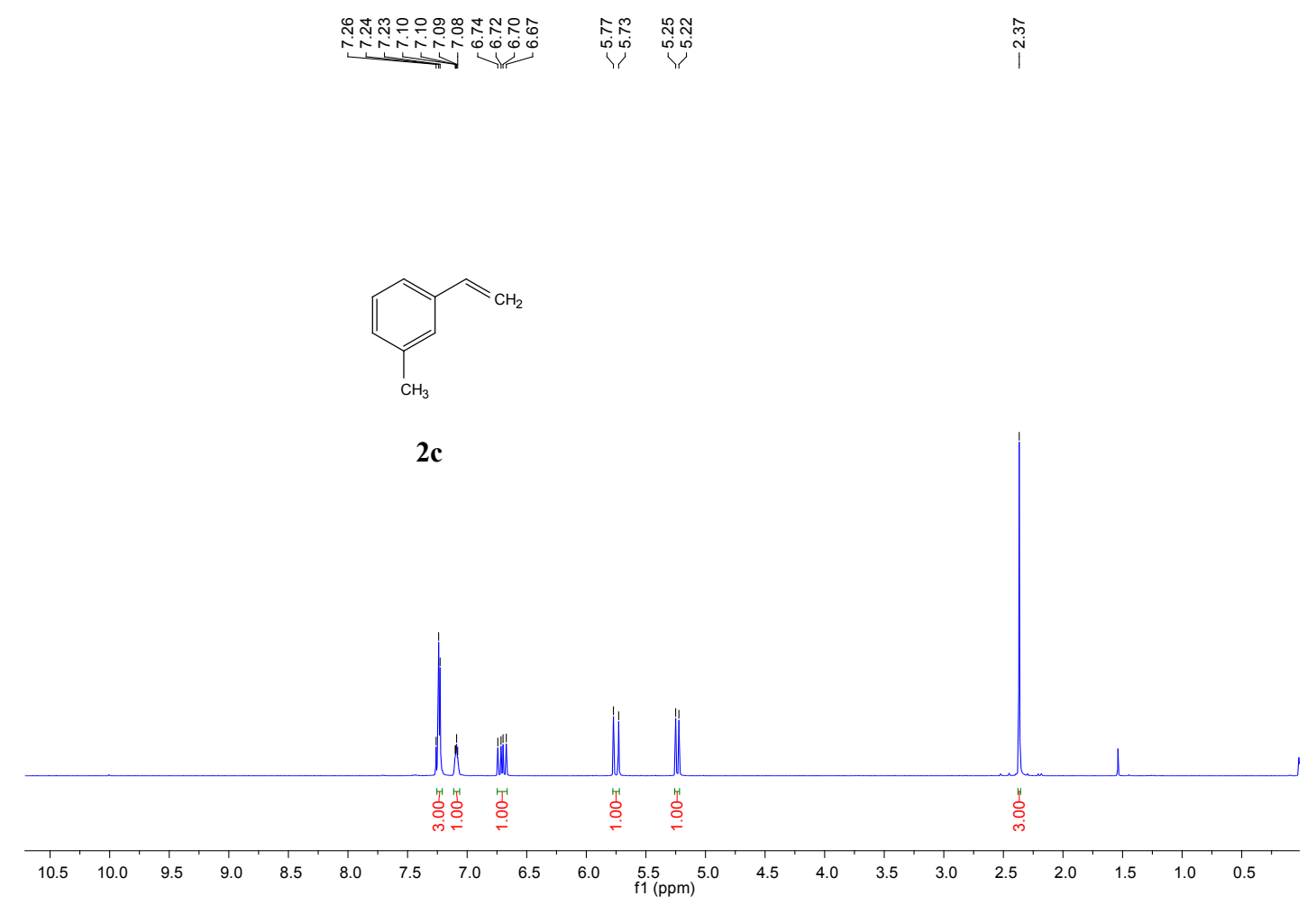

2c

Figure S6. ${ }^{13} \mathrm{C}$ NMR spectrum $\left(100 \mathrm{MHz}, \mathrm{CDCl}_{3}\right)$ of compound $2 \mathrm{c}$

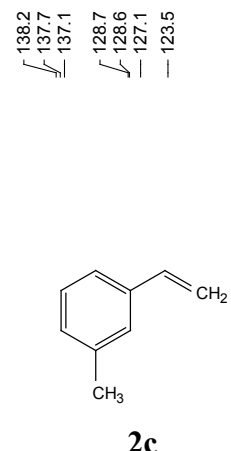

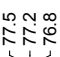

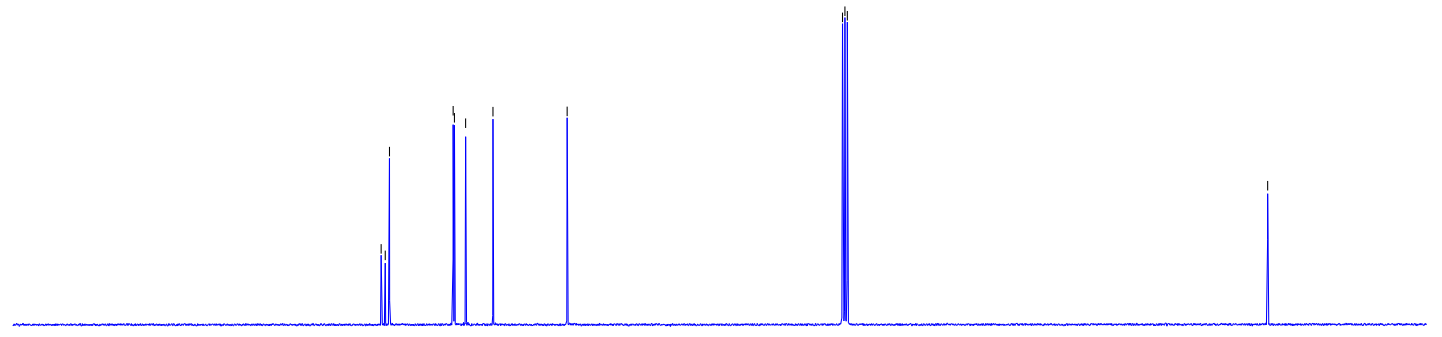

$\begin{array}{lllllllll}180 & 170 & 160 & 150 & 140 & 130 & 120 & 110 & 100 \\ \mathrm{f} 1(\mathrm{ppm}) & 90\end{array}$

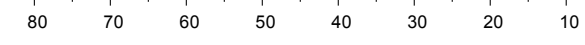


Figure S7. ${ }^{1} \mathrm{H}$ NMR spectrum (400 $\mathrm{MHz}^{\mathrm{CDCl}_{3}}$, TMS) of compound 2d

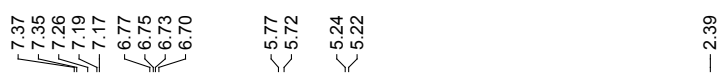

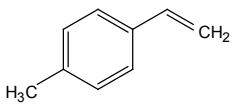

2d

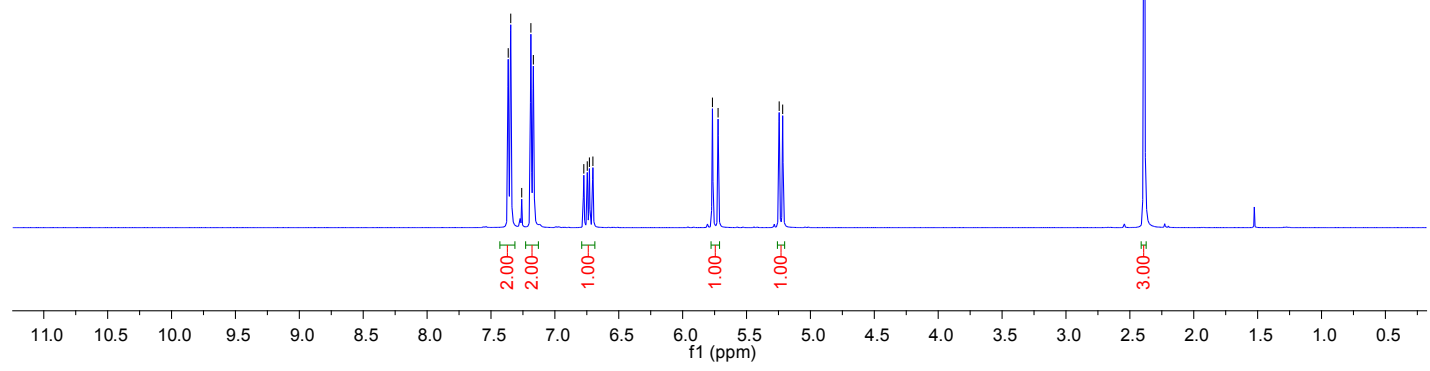

Figure S8. ${ }^{13} \mathrm{C}$ NMR spectrum $\left(100 \mathrm{MHz}, \mathrm{CDCl}_{3}\right)$ of compound $2 \mathrm{~d}$

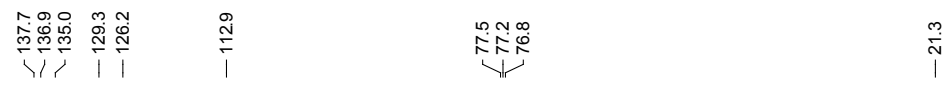

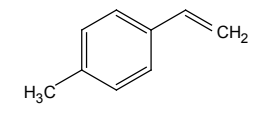

2d

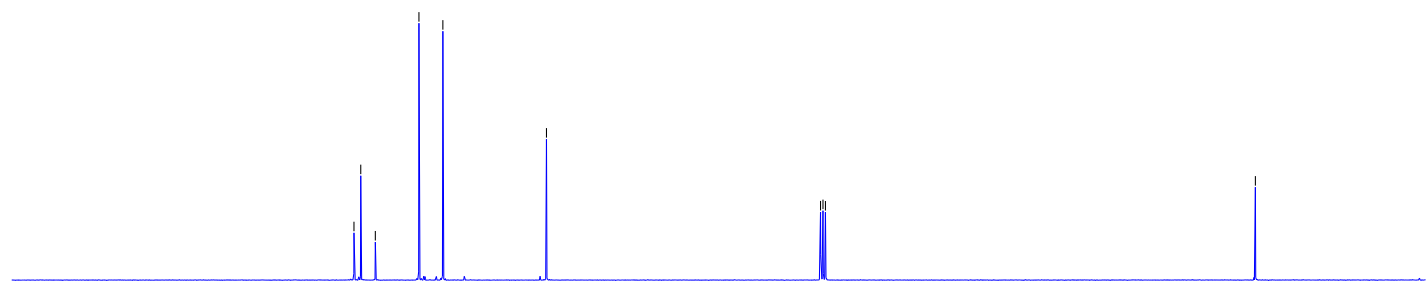

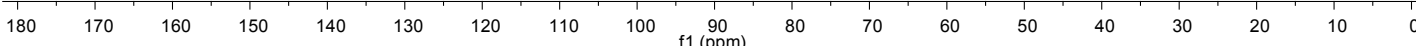


Figure S9. ${ }^{1} \mathrm{H}$ NMR spectrum (400 MHz, $\mathrm{CDCl}_{3}$, TMS) of compound $2 \mathrm{e}$

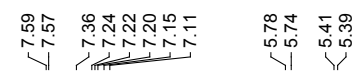

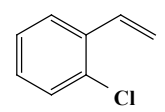

$2 e$

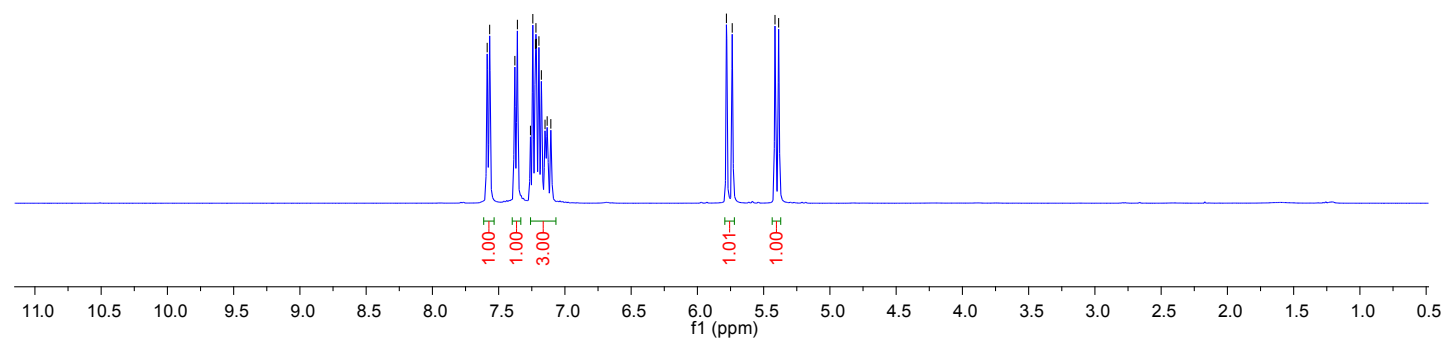

Figure S10. ${ }^{13} \mathrm{C}$ NMR spectrum (100 MHz, $\left.\mathrm{CDCl}_{3}\right)$ of compound 2e
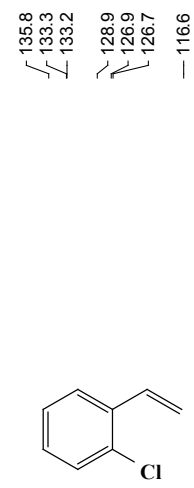

$2 \mathbf{e}$

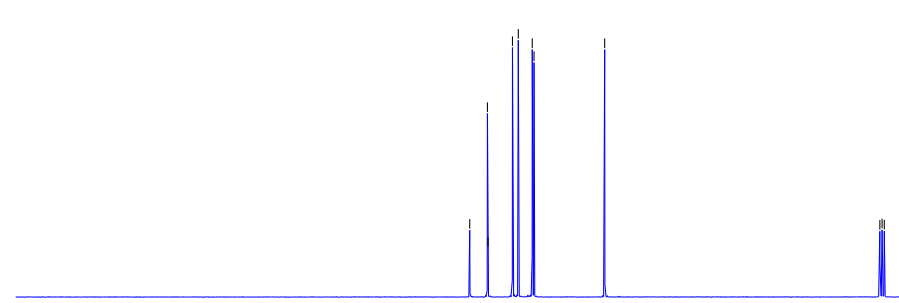

200

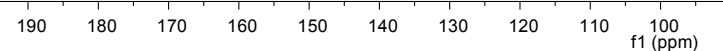
$\begin{array}{llll}90 & 80 & 70 & 60\end{array}$ 
Figure S11. ${ }^{1} \mathrm{H}$ NMR spectrum (400 $\mathrm{MHz}, \mathrm{CDCl}_{3}$, TMS) of compound $2 \mathrm{f}$

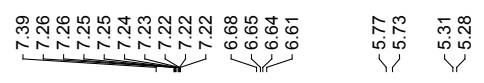

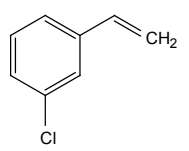

2f

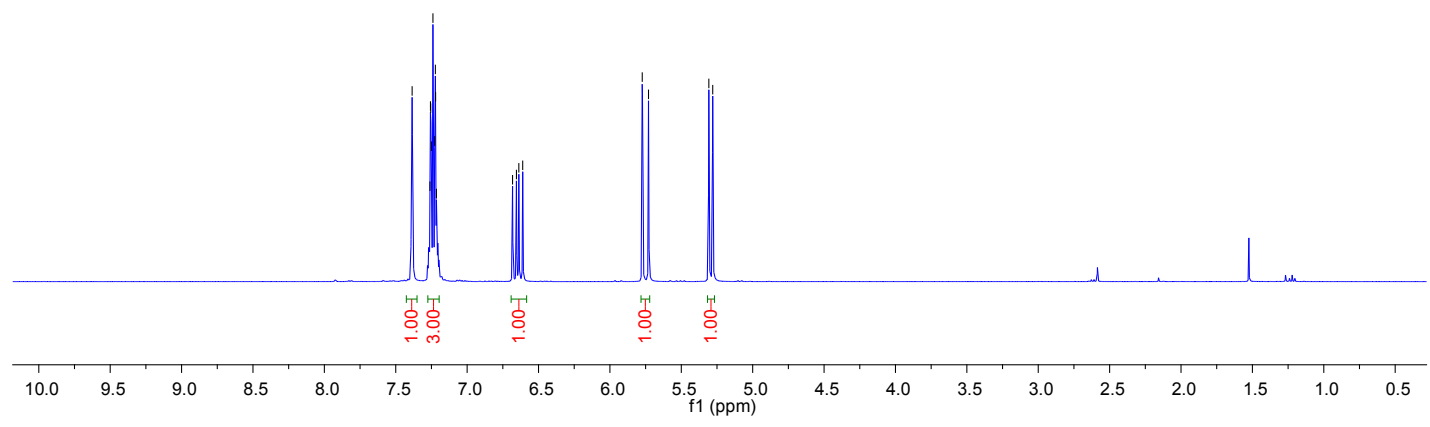

Figure S12. ${ }^{13} \mathrm{C}$ NMR spectrum $\left(100 \mathrm{MHz} \mathrm{CDCl}_{3}\right)$ of compound $2 \mathrm{f}$

$$
\text { l }
$$

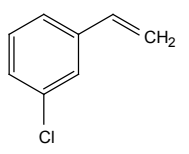

2f

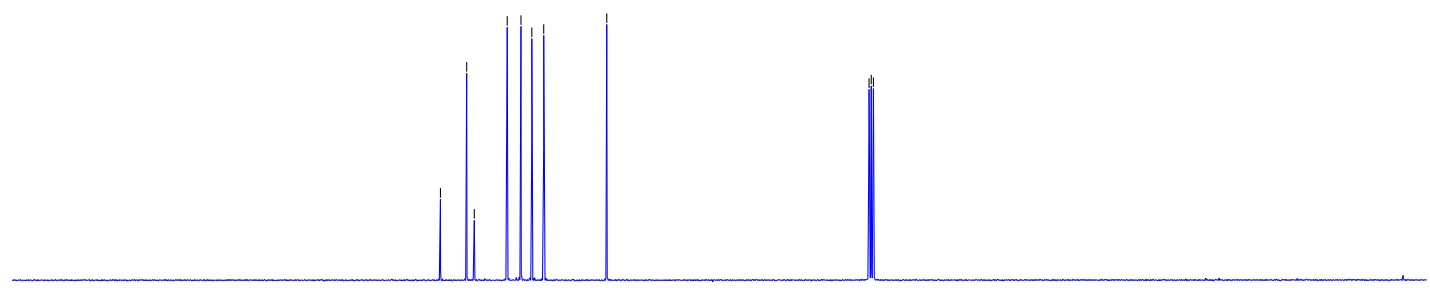

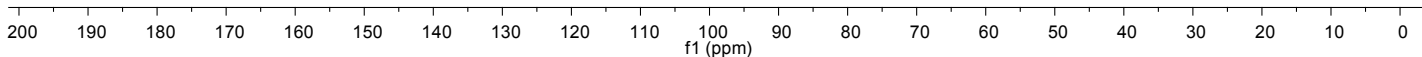


Figure S13. ${ }^{1} \mathrm{H}$ NMR spectrum (400 $\mathrm{MHz}, \mathrm{CDCl}_{3}$, TMS) of compound $2 \mathrm{~g}$

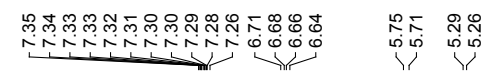

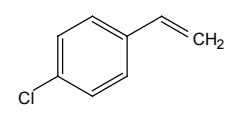

$2 \mathrm{~g}$

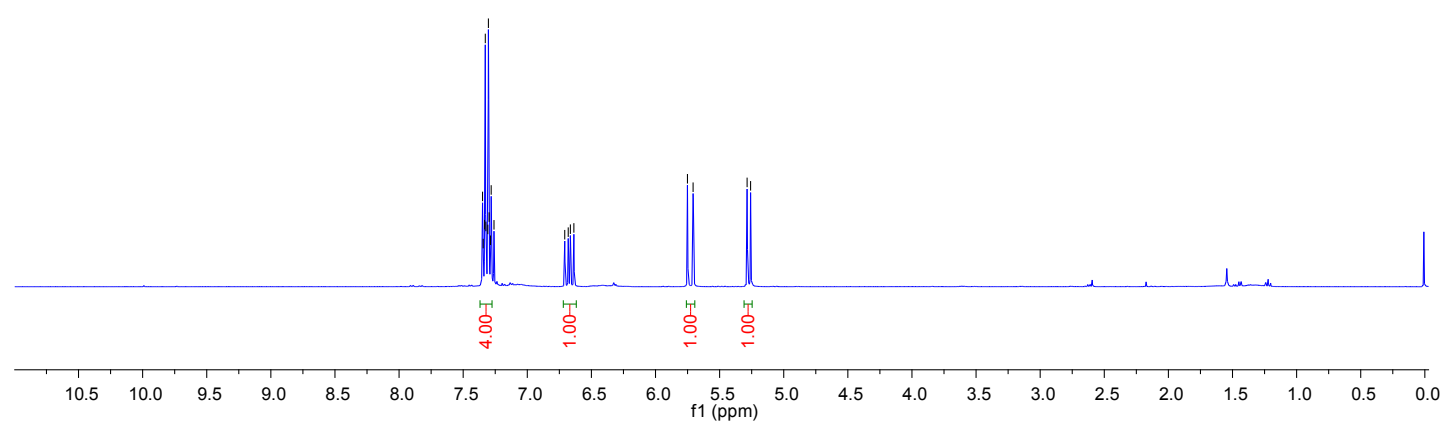

Figure S14. ${ }^{13} \mathrm{C}$ NMR spectrum $\left(100 \mathrm{MHz}, \mathrm{CDCl}_{3}\right)$ of compound $2 \mathrm{~g}$
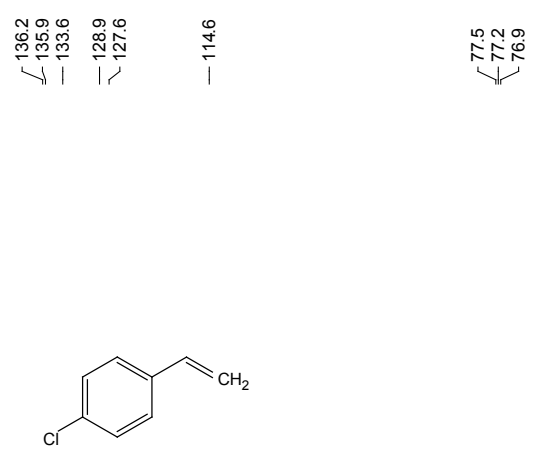

$2 \mathrm{~g}$

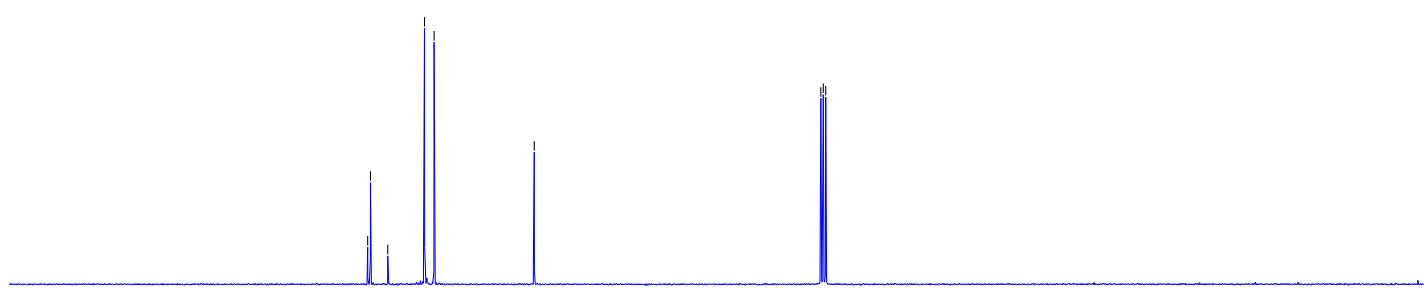

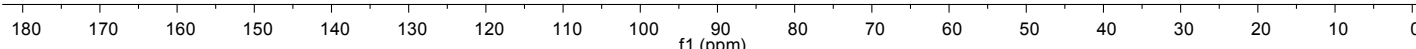


Figure S15. ${ }^{1} \mathrm{H}$ NMR spectrum (400 $\mathrm{MHz}, \mathrm{CDCl}_{3}$, TMS) of compound $2 \mathrm{~h}$

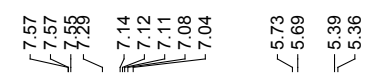

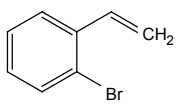

2h

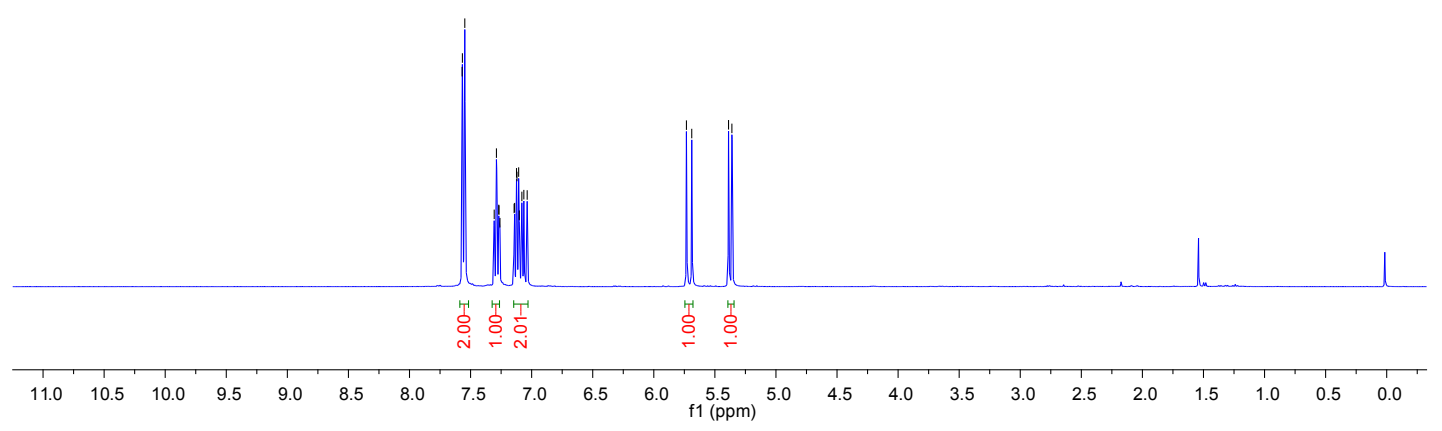

Figure S16. ${ }^{13} \mathrm{C}$ NMR spectrum (100 $\left.\mathrm{MHz} \mathrm{CDCl}_{3}\right)$ of compound $2 \mathrm{~h}$

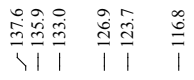

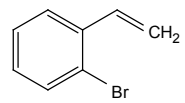

$2 \mathbf{h}$

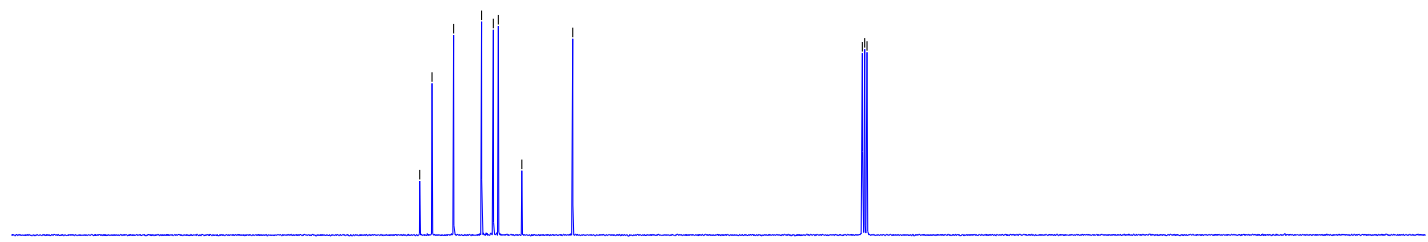

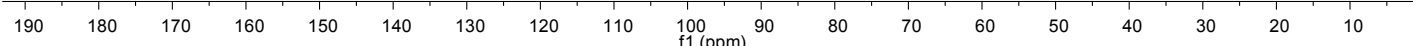


Figure S17. ${ }^{1} \mathrm{H}$ NMR spectrum (400 $\mathrm{MHz}, \mathrm{CDCl}_{3}$, TMS) of compound $2 \mathrm{i}$

11

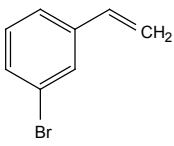

$2 \mathrm{i}$

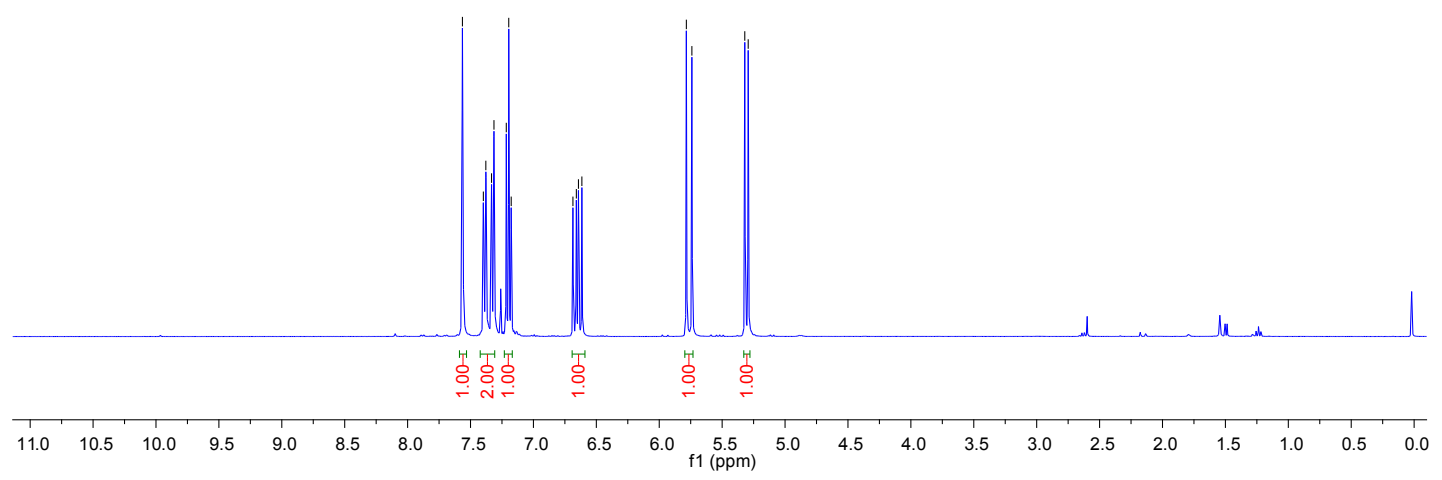

Figure S18. ${ }^{13} \mathrm{C}$ NMR spectrum (100 $\left.\mathrm{MHz}, \mathrm{CDCl}_{3}\right)$ of compound $2 \mathrm{i}$

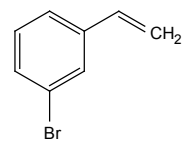

$2 i$

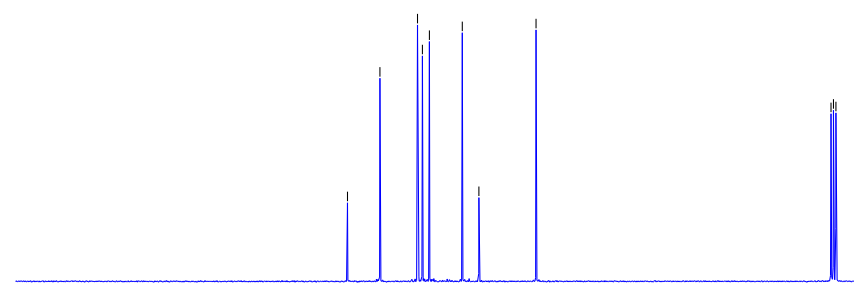

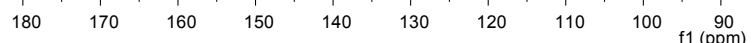

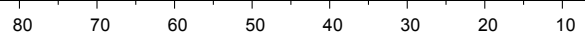


Figure S19. ${ }^{1} \mathrm{H}$ NMR spectrum (400 $\mathrm{MHz}, \mathrm{CDCl}_{3}$, TMS) of compound $2 \mathrm{j}$
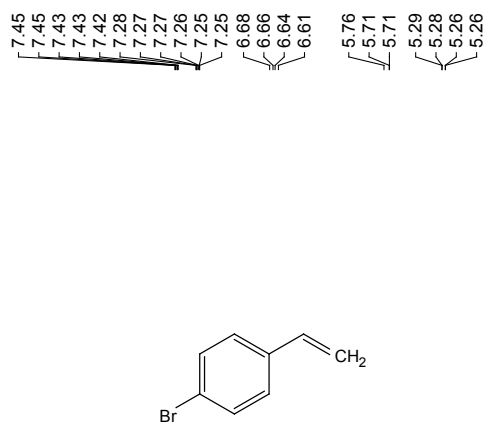

$2 j$

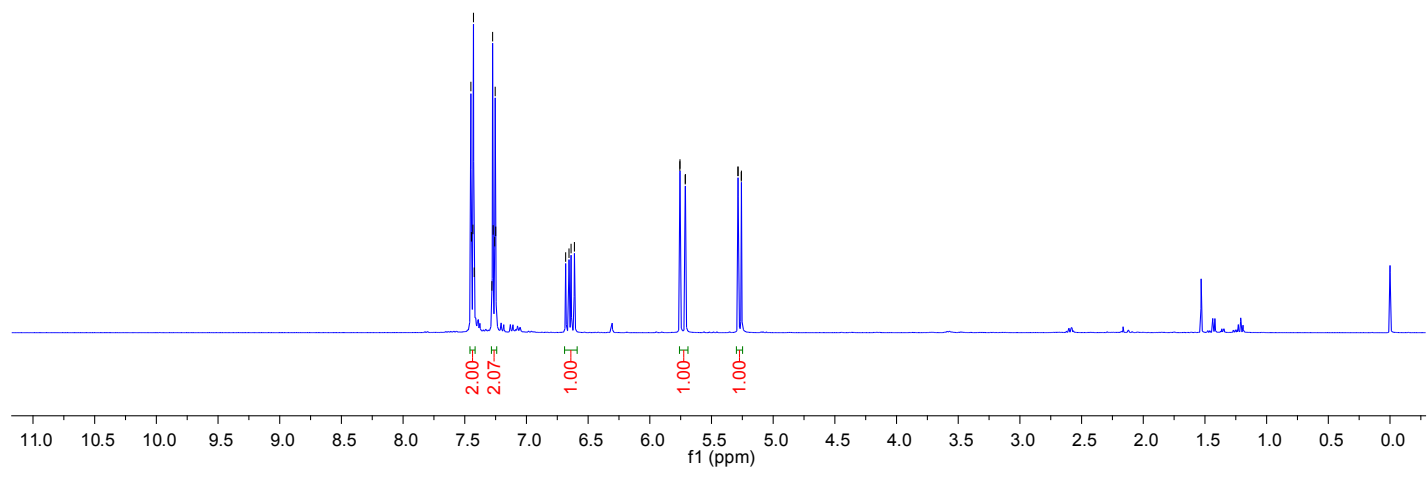

Figure S20. ${ }^{13} \mathrm{C}$ NMR spectrum $\left(100 \mathrm{MHz}, \mathrm{CDCl}_{3}\right)$ of compound $2 \mathrm{j}$

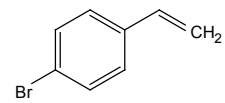

$2 \mathbf{j}$

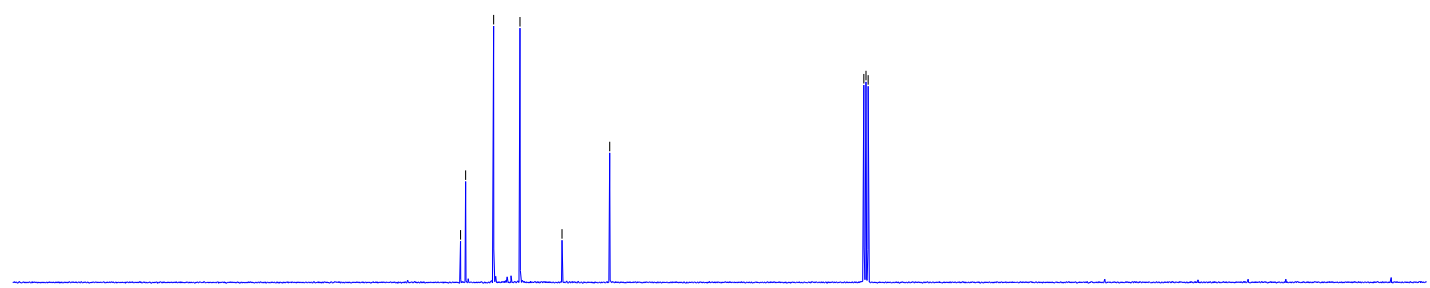

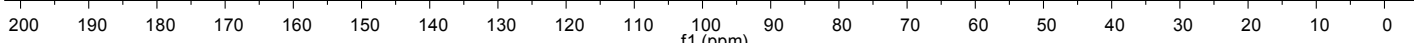


Figure S21. ${ }^{1} \mathrm{H}$ NMR spectrum (400 $\mathrm{MHz}^{\mathrm{CDCl}} \mathrm{CDMS}_{3}$, TM of compound $2 \mathrm{k}$

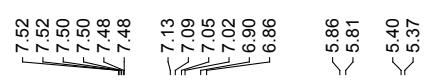

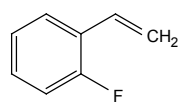

$2 k$

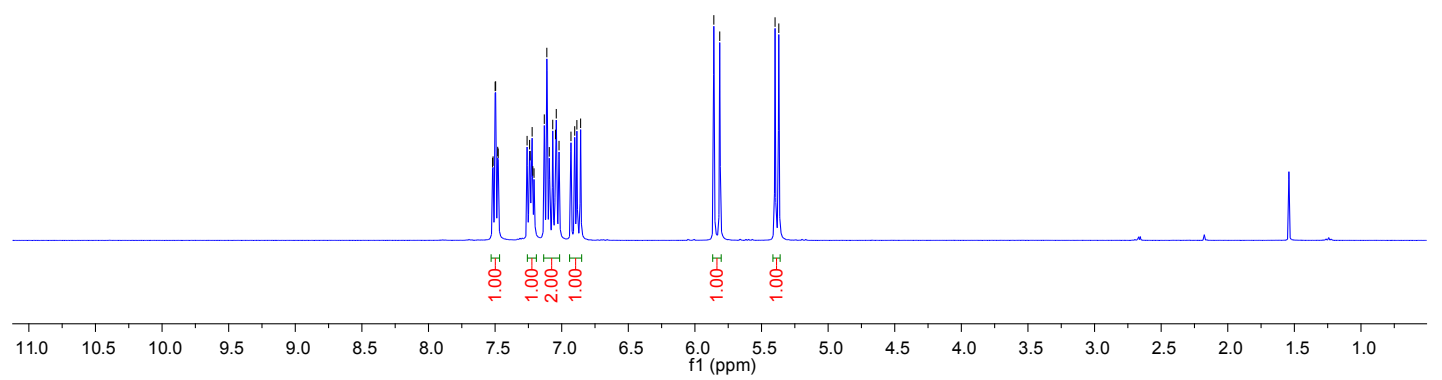

Figure S22. ${ }^{13} \mathrm{C}$ NMR spectrum $\left(100 \mathrm{MHz}, \mathrm{CDCl}_{3}\right)$ of compound $2 \mathrm{k}$
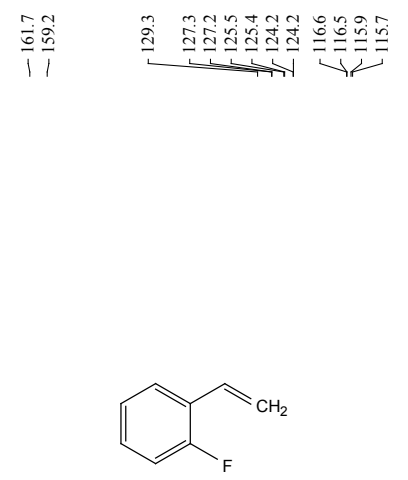

2k

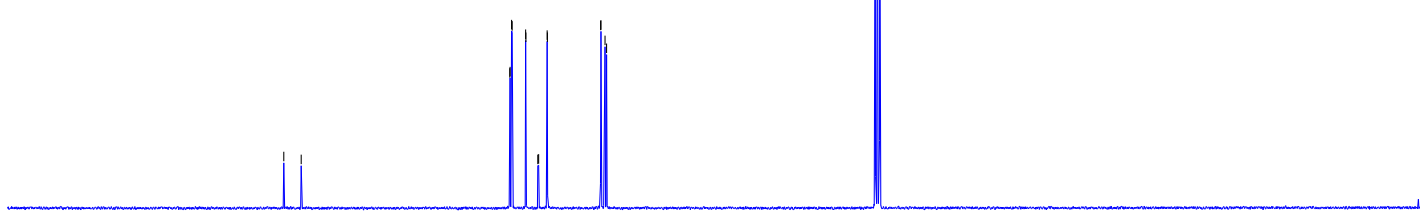

$\begin{array}{llllllllllllllllllllll}1 & 100 & 190 & 180 & 170 & 160 & 150 & 140 & 130 & 120 & 110 & 100 & 90 & 80 & 70 & 60 & 50 & 40 & 30 & 20 & 10\end{array}$ 
Figure S23. ${ }^{1} \mathrm{H}$ NMR spectrum (400 $\mathrm{MHz}, \mathrm{CDCl}_{3}$, TMS) of compound 21

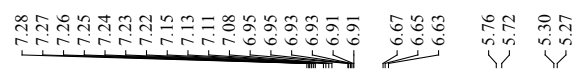

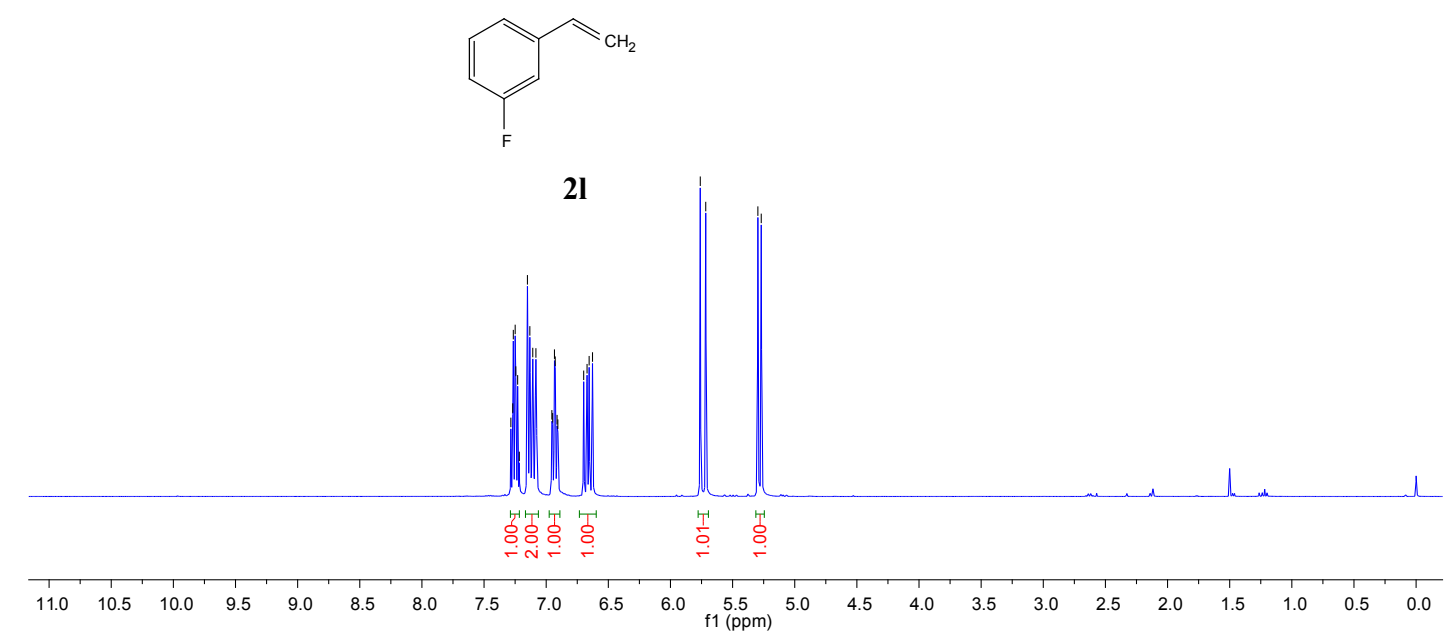

Figure S24. ${ }^{13} \mathrm{C}$ NMR spectrum $\left(100 \mathrm{MHz}, \mathrm{CDCl}_{3}\right)$ of compound $2 \mathrm{l}$

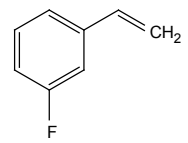

21

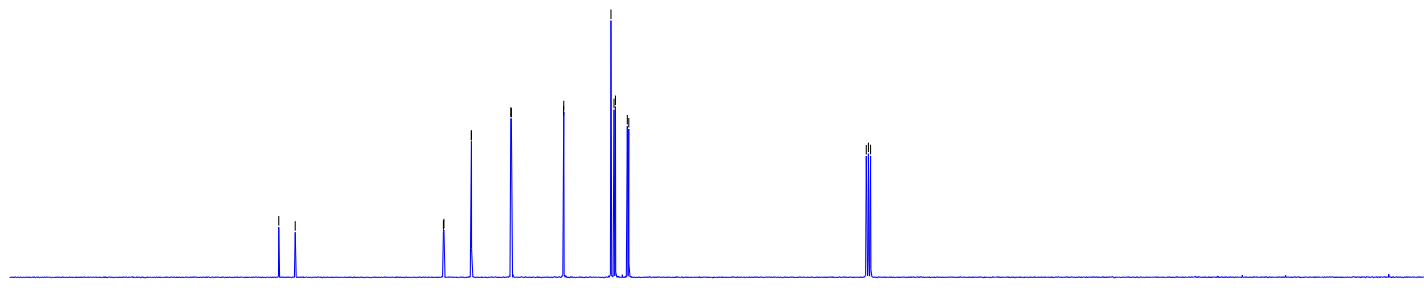

$\begin{array}{llllllllllllllllllllll}1 & 100 & 190 & 180 & 170 & 160 & 150 & 140 & 130 & 120 & 110 & 100 & 90 & 80 & 70 & 60 & 50 & 40 & 30 & 20 & 10 & 0\end{array}$ 
Figure S25. ${ }^{1} \mathrm{H}$ NMR spectrum (400 $\mathrm{MHz}^{\mathrm{CDCl}_{3}}$, TMS) of compound $2 \mathrm{~m}$
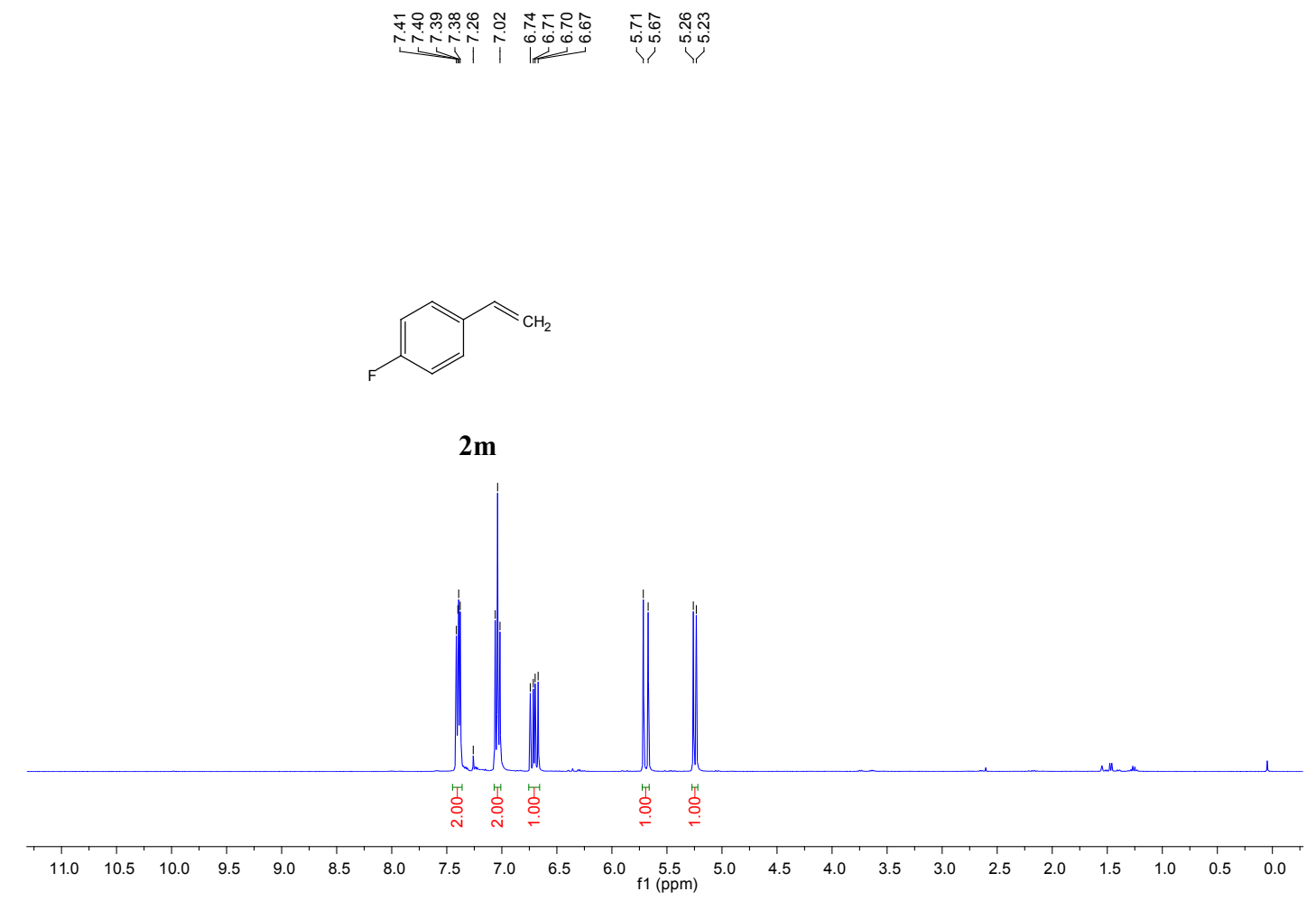

Figure S26. ${ }^{13} \mathrm{C}$ NMR spectrum (100 $\left.\mathrm{MHz}, \mathrm{CDCl}_{3}\right)$ of compound $2 \mathrm{~m}$

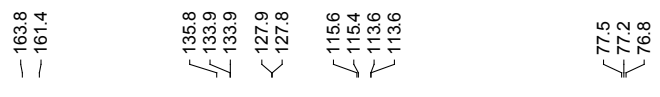<smiles>C=Cc1ccc(F)cc1</smiles>

$2 \mathbf{m}$

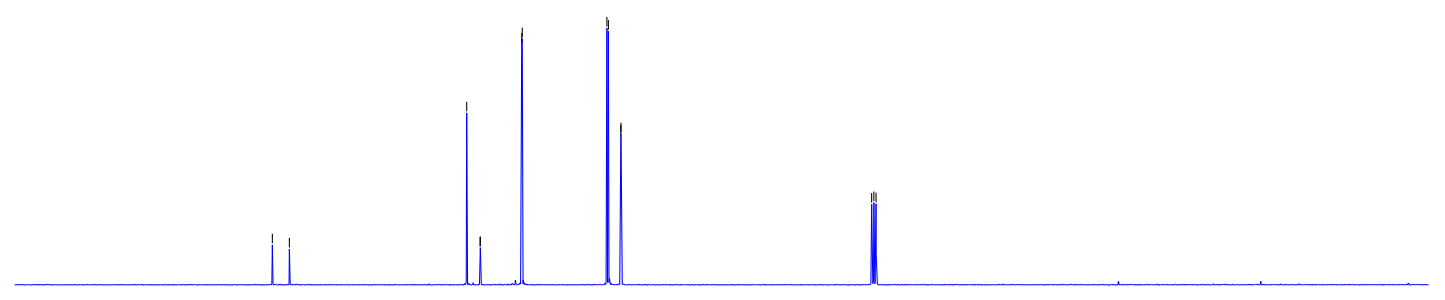

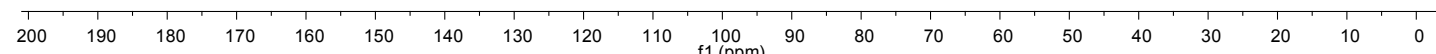


Figure S27. ${ }^{1} \mathrm{H}$ NMR spectrum (400 $\mathrm{MHz}, \mathrm{CDCl}_{3}$, TMS) of compound 2n

لi

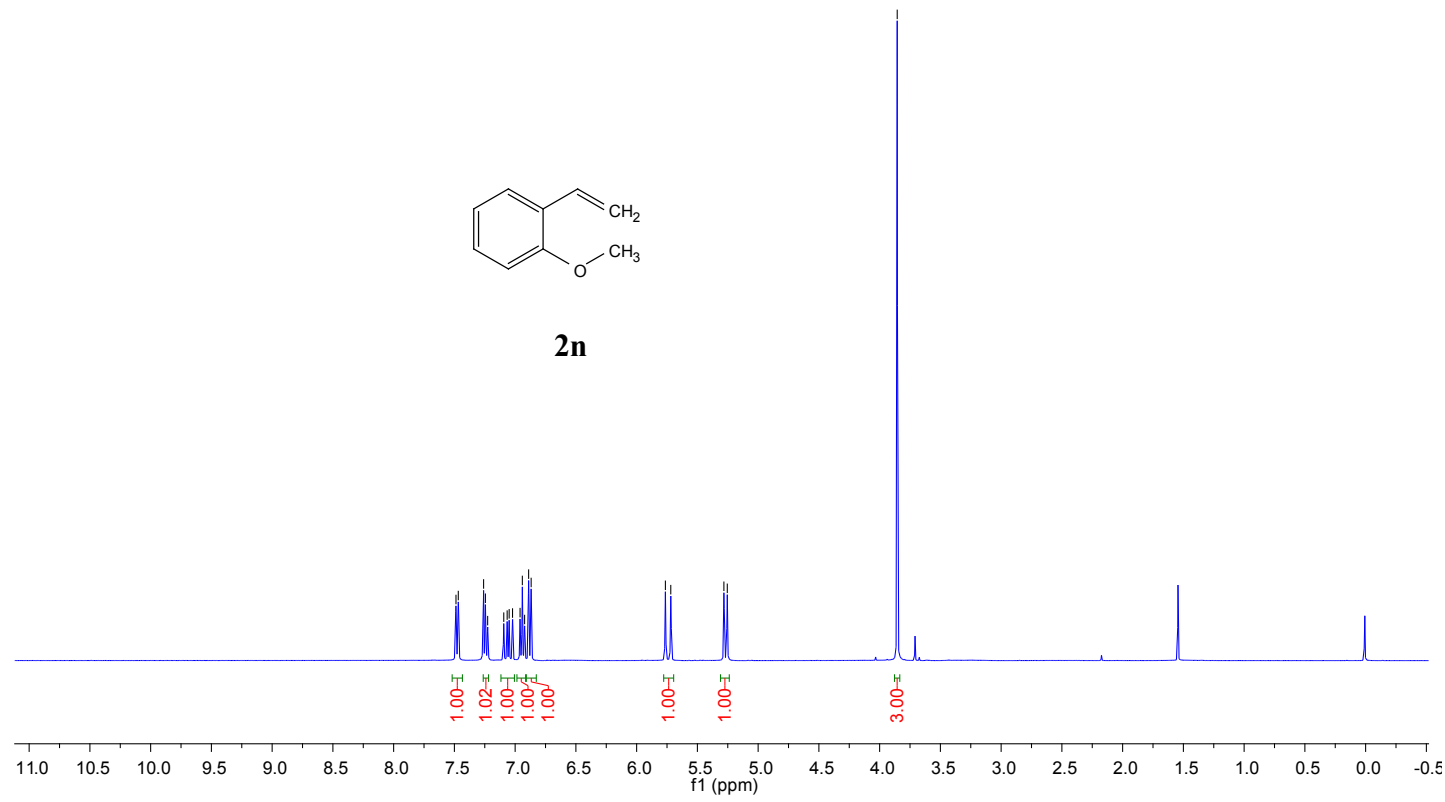

Figure S28. ${ }^{13} \mathrm{C}$ NMR spectrum (100 $\left.\mathrm{MHz} \mathrm{CDCl}_{3}\right)$ of compound 2n

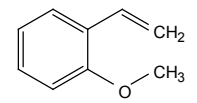

2n

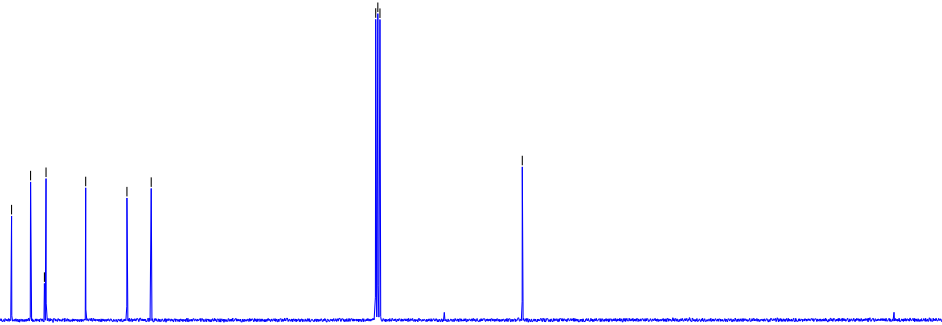

$\begin{array}{llllllllllll}200 & 190 & 180 & 170 & 160 & 150 & 140 & 130 & 120 & 110 & \begin{array}{l}100 \\ \mathrm{f} 1(\mathrm{ppm})\end{array} & 90\end{array}$

$70 \quad 60$

$\begin{array}{llllll}50 & 40 & 30 & 20 & 10 & 0\end{array}$ 
Figure S29. ${ }^{1} \mathrm{H}$ NMR spectrum (400 $\mathrm{MHz}, \mathrm{CDCl}_{3}$, TMS) of compound 20
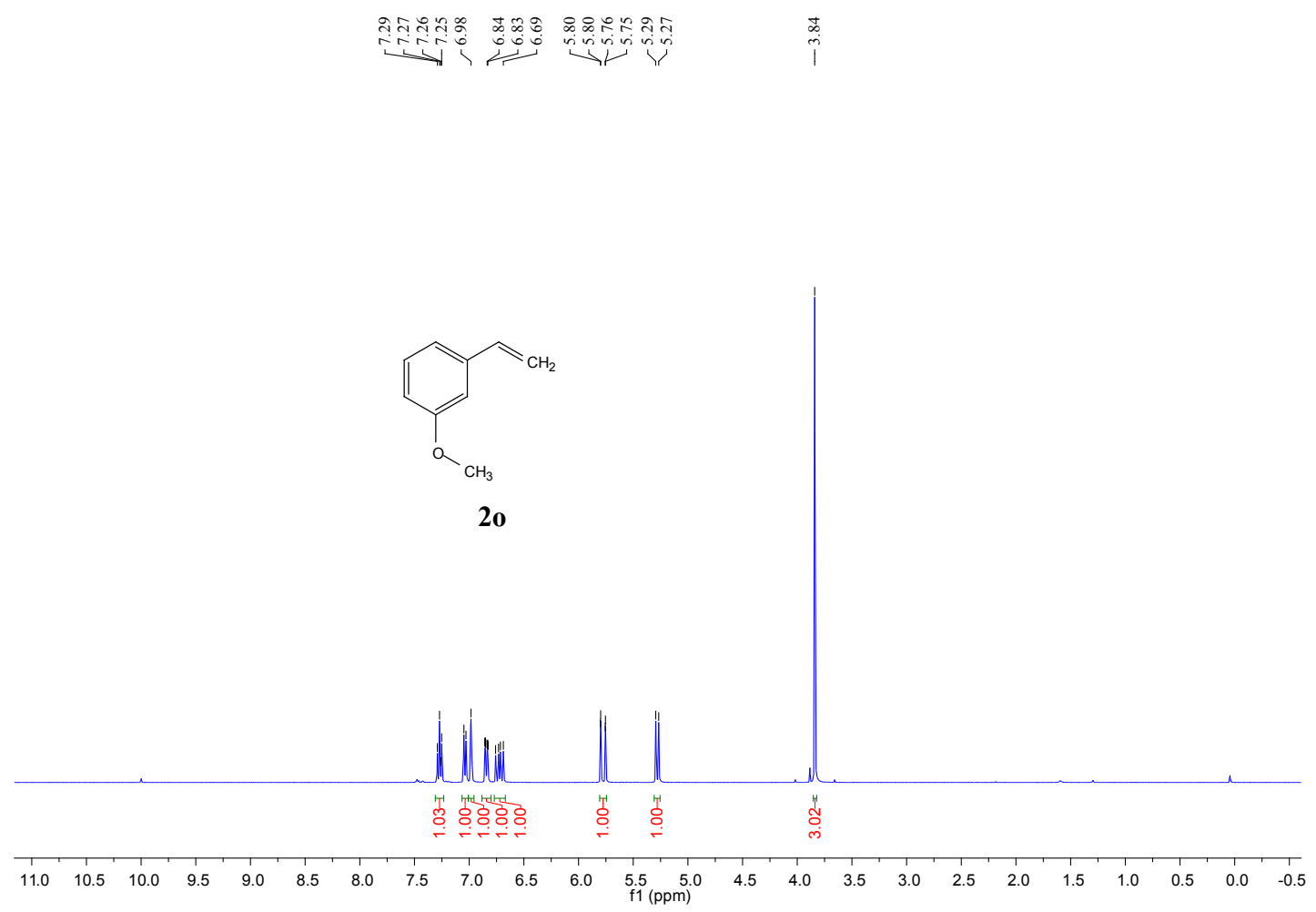

Figure S30. ${ }^{13} \mathrm{C}$ NMR spectrum (100 $\left.\mathrm{MHz}, \mathrm{CDCl}_{3}\right)$ of compound 20

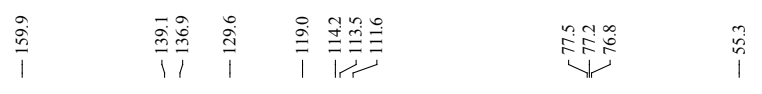

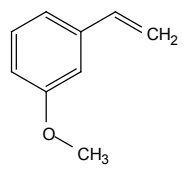

20

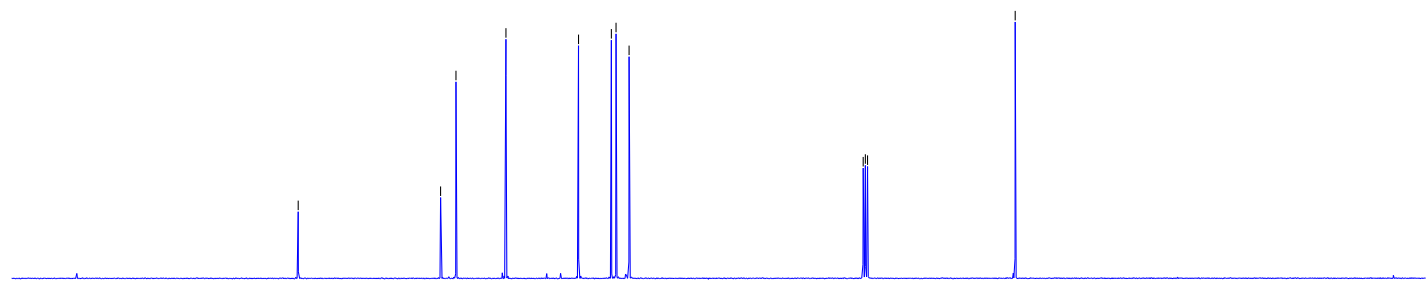

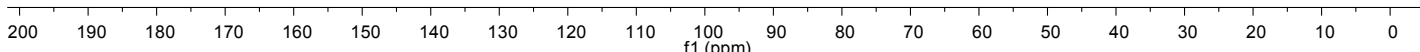


Figure S31. ${ }^{1} \mathrm{H}$ NMR spectrum (400 $\mathrm{MHz}, \mathrm{CDCl}_{3}$, TMS) of compound 2p
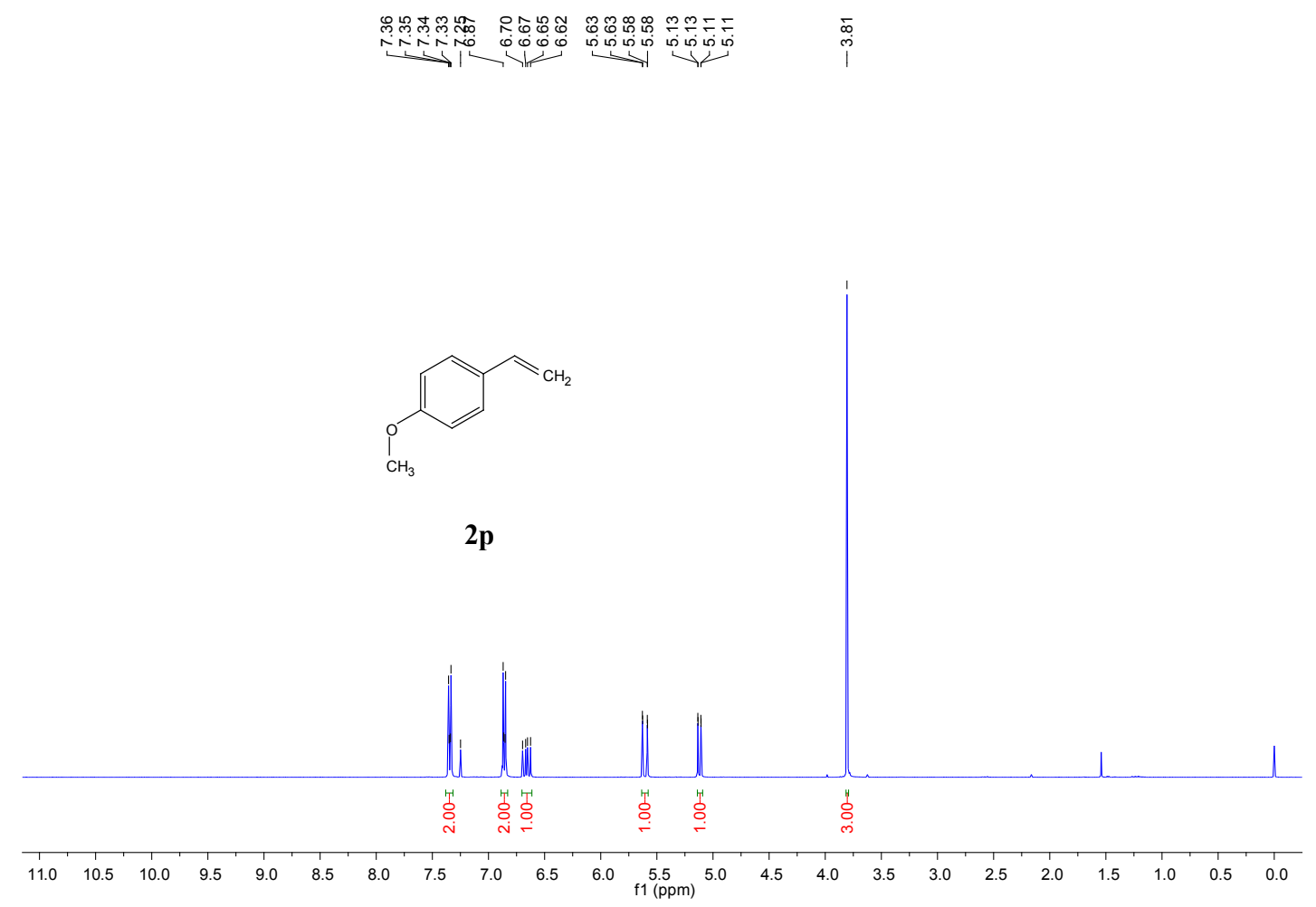

$2 p$

$\underset{\infty}{\infty}$

Figure S32. ${ }^{13} \mathrm{C}$ NMR spectrum (100 $\left.\mathrm{MHz}, \mathrm{CDCl}_{3}\right)$ of compound 2p

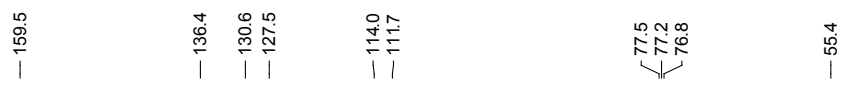

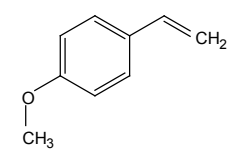

$2 \mathbf{p}$

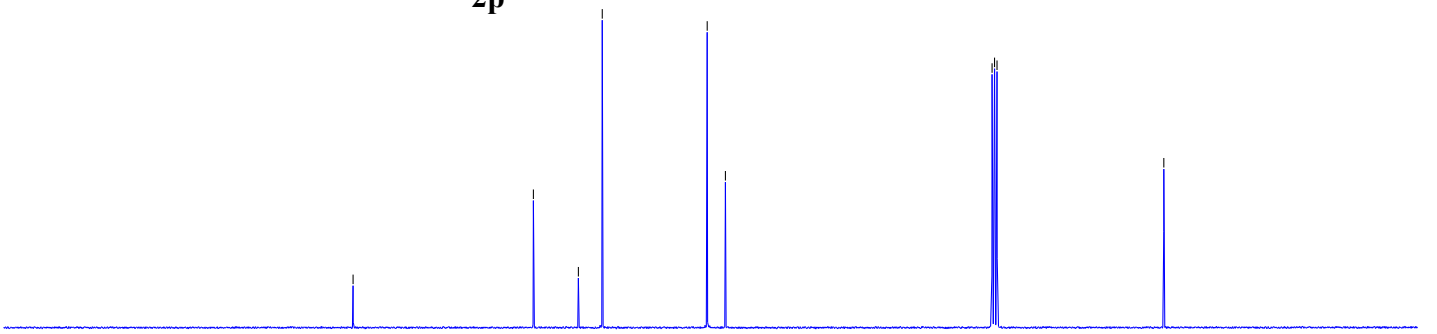

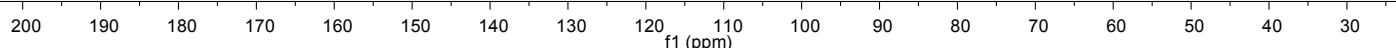


Figure S33. ${ }^{1} \mathrm{H}$ NMR spectrum (400 $\mathrm{MHz} \mathrm{CDCl}_{3}$, TMS) of compound $2 \mathrm{q}$

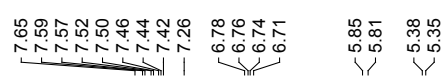

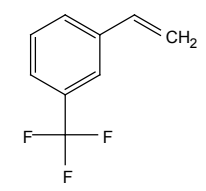

$\mathbf{2 q}$

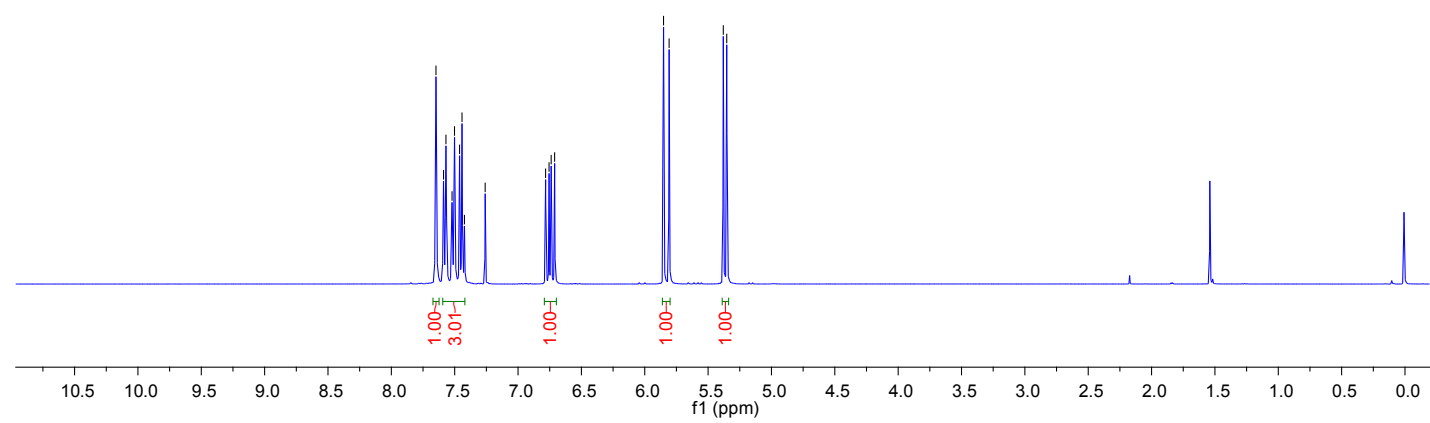

Figure S34. ${ }^{13} \mathrm{C}$ NMR spectrum (100 $\left.\mathrm{MHz} \mathrm{CDCl}_{3}\right)$ of compound $2 \mathrm{q}$

$$
\underset{1}{1}
$$

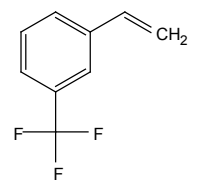

$2 q$

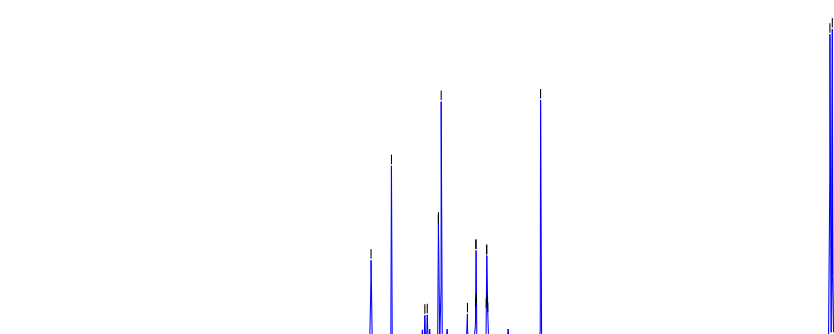

$\begin{array}{lllllllll}180 & 170 & 160 & 150 & 140 & 130 & 120 & 110 & 100 \begin{array}{r}90 \\ \mathrm{f} 1(\mathrm{ppm})\end{array}\end{array}$ 
Figure S35. ${ }^{1} \mathrm{H}$ NMR spectrum (400 $\mathrm{MHz}, \mathrm{CDCl}_{3}$, TMS) of compound 2r
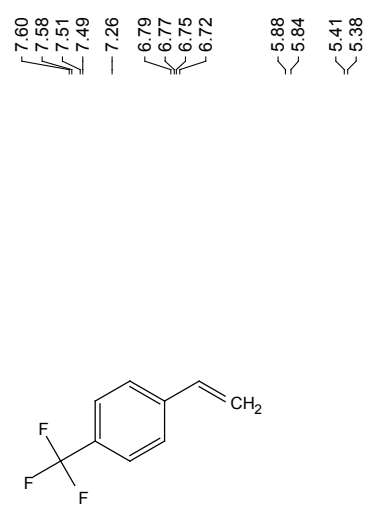

$2 r$

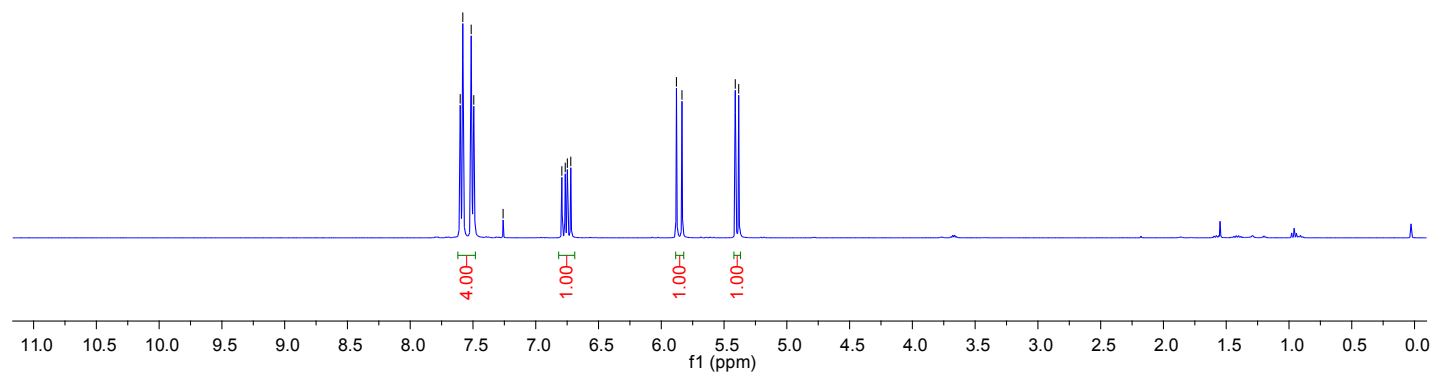

Figure S36. ${ }^{13} \mathrm{C}$ NMR spectrum (100 $\left.\mathrm{MHz}^{\mathrm{CDCl}}\right)_{3}$ of compound 2r
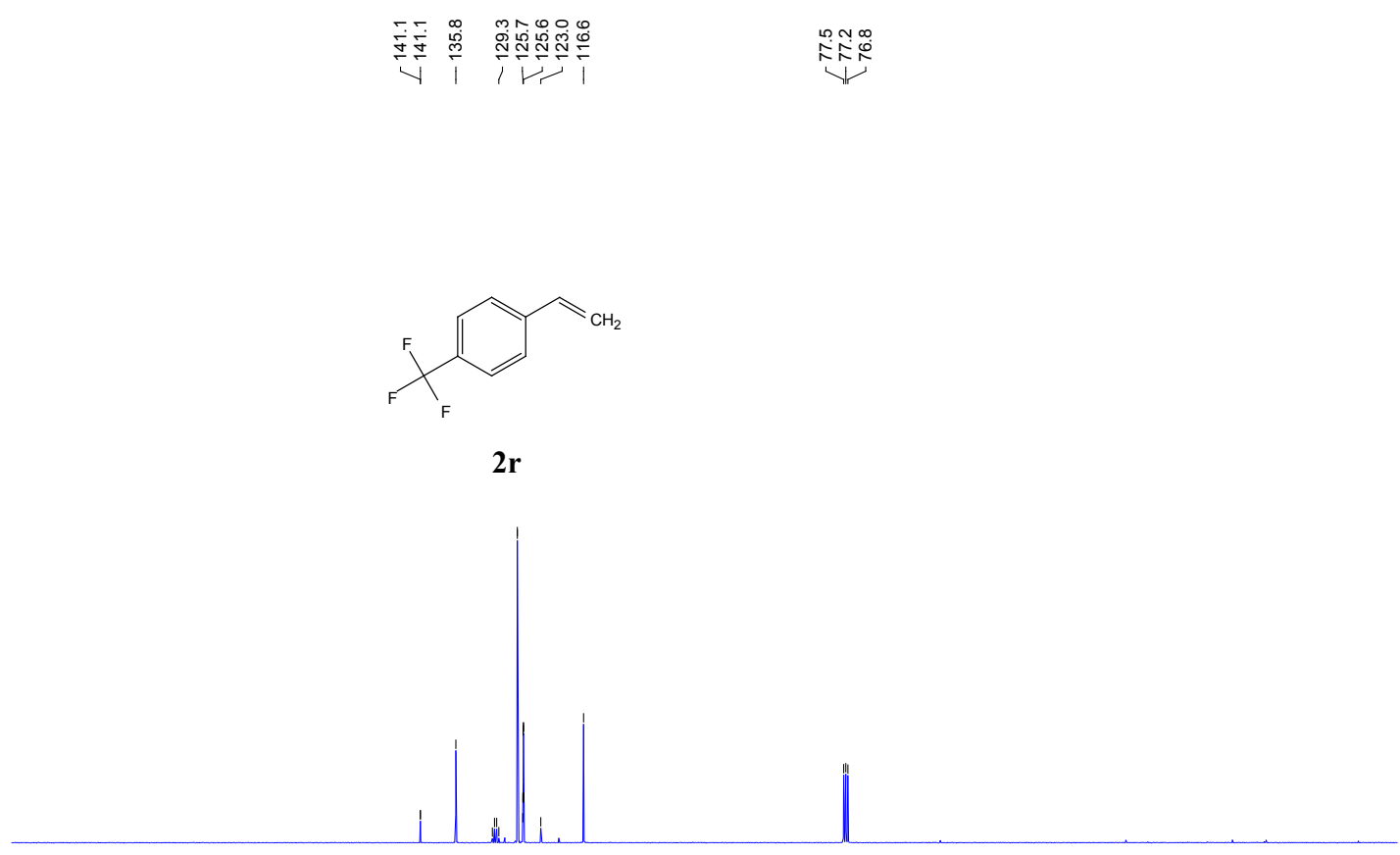

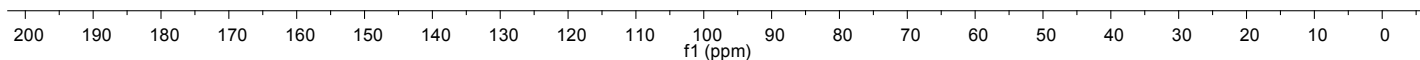


Figure S37. ${ }^{1} \mathrm{H}$ NMR spectrum (400 $\mathrm{MHz}, \mathrm{CDCl}_{3}$, TMS) of compound $2 \mathrm{~s}$
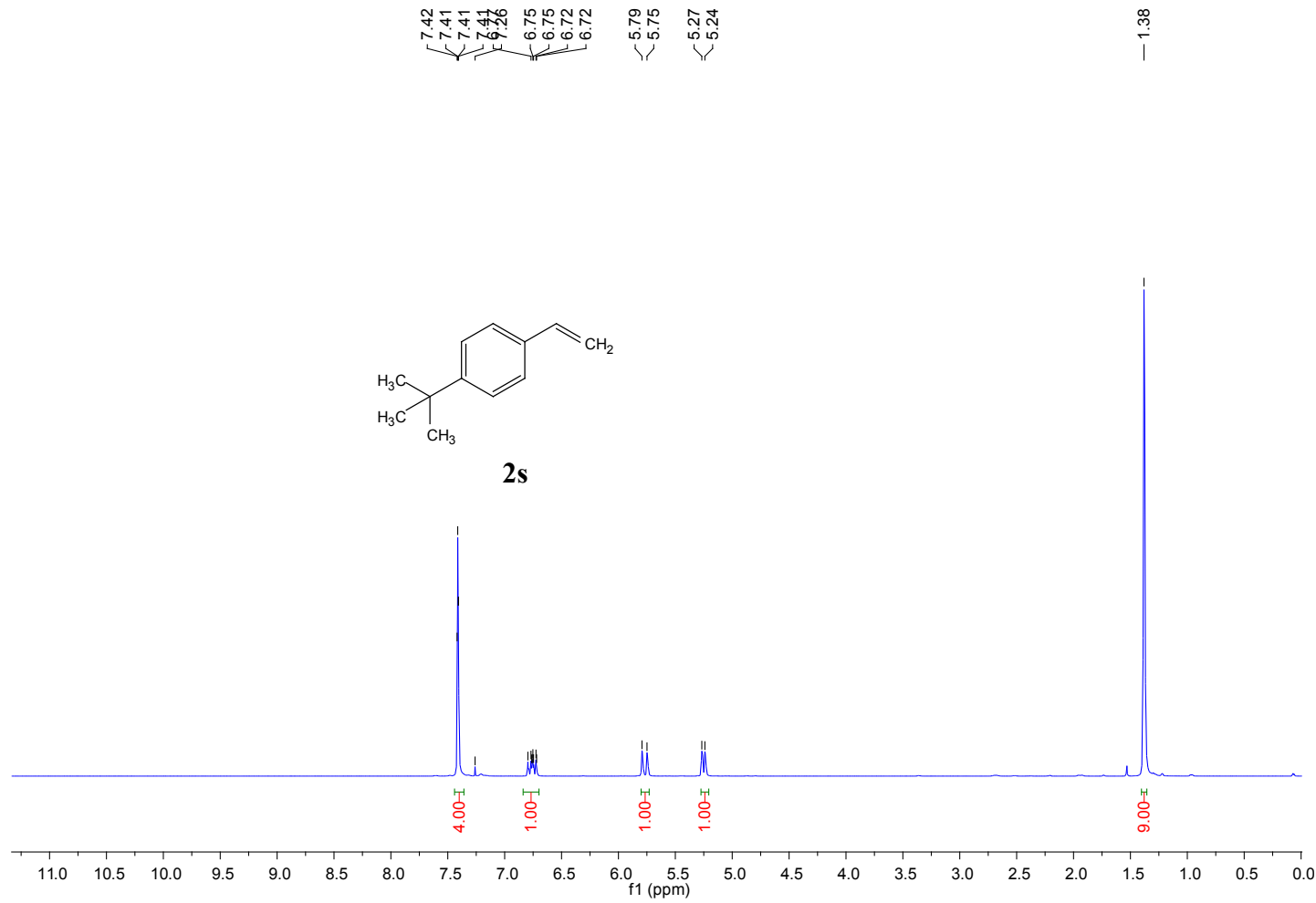

Figure S38. ${ }^{13} \mathrm{C}$ NMR spectrum $\left(100 \mathrm{MHz} \mathrm{CDCl}_{3}\right)$ of compound $2 \mathrm{~s}$
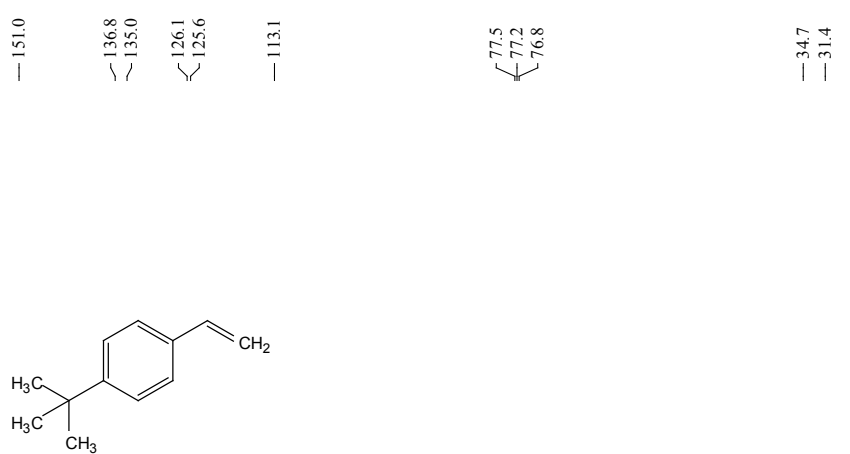

$2 s$

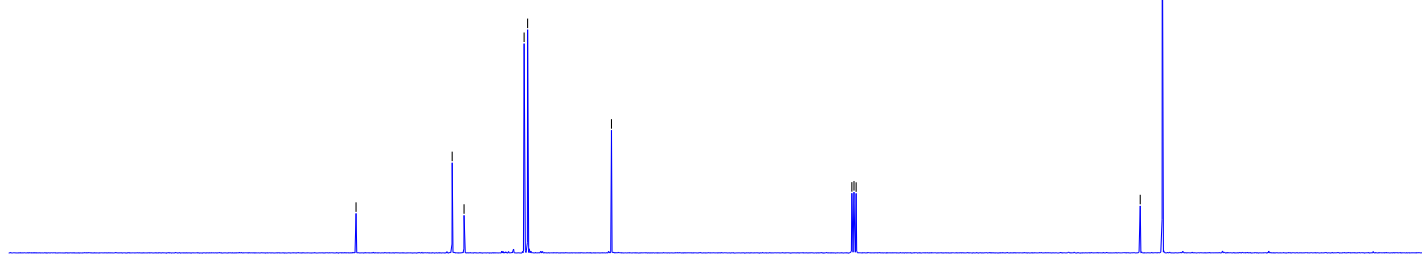

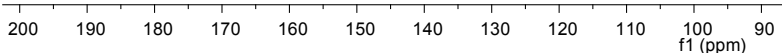


Figure S39. ${ }^{1} \mathrm{H}$ NMR spectrum (400 $\mathrm{MHz}, \mathrm{CDCl}_{3}$, TMS) of compound $2 \mathrm{t}$
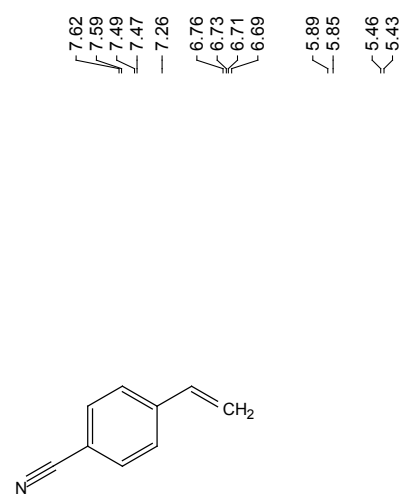

$2 t$

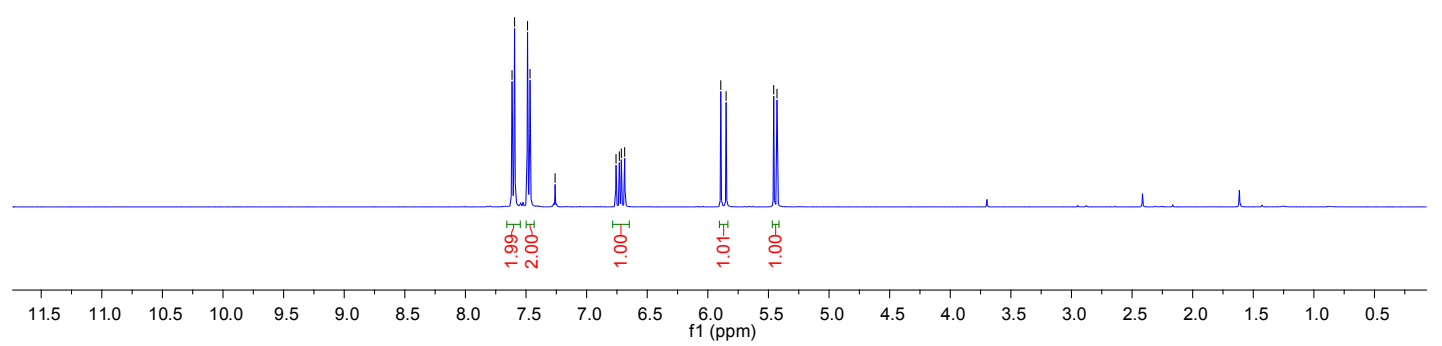

Figure S40. ${ }^{13} \mathrm{C}$ NMR spectrum $\left(100 \mathrm{MHz} \mathrm{CDCl}_{3}\right)$ of compound $2 \mathrm{t}$
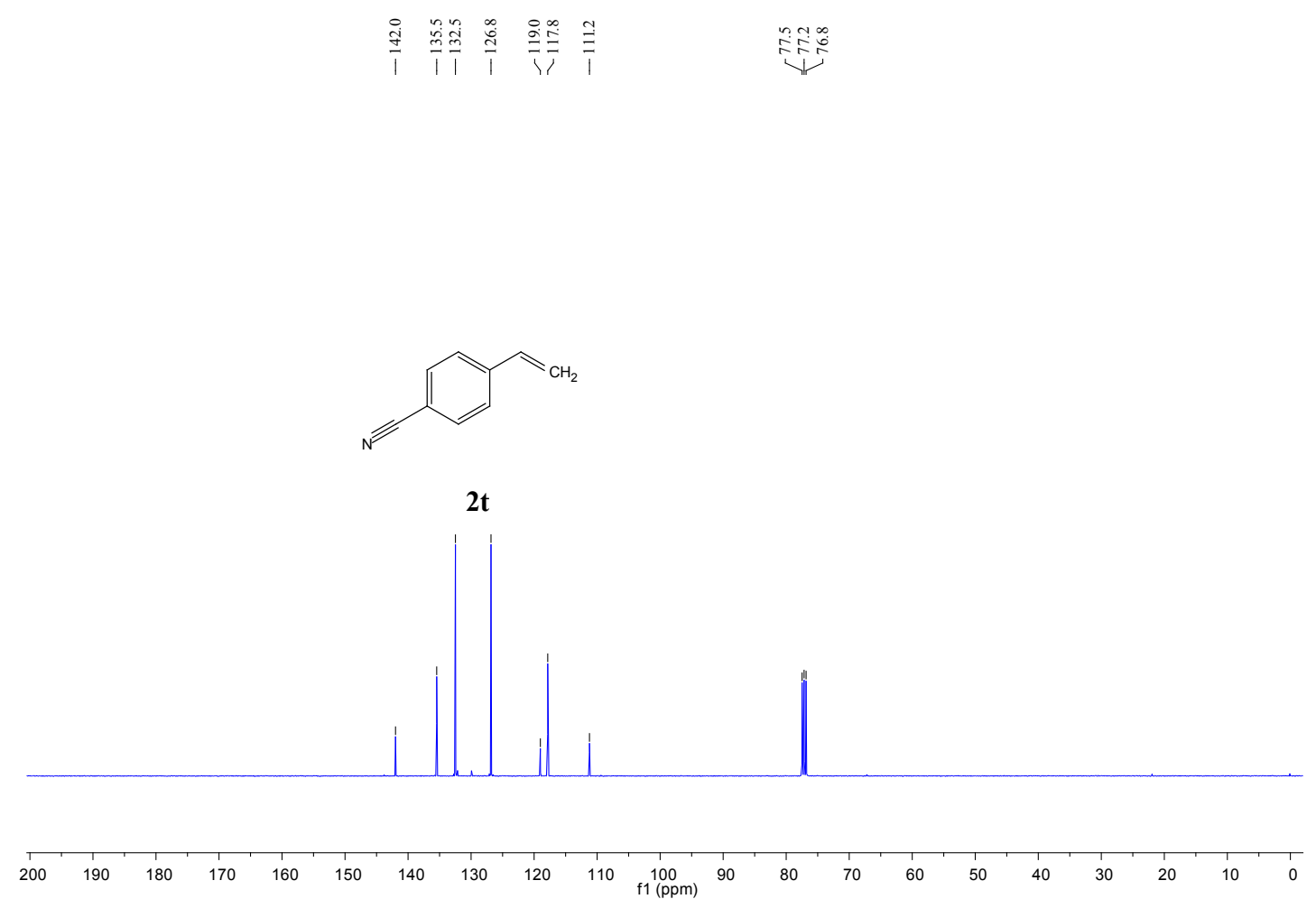
Figure S41. ${ }^{1} \mathrm{H}$ NMR spectrum (400 $\mathrm{MHz}, \mathrm{CDCl}_{3}$, TMS) of compound $2 \mathrm{u}$

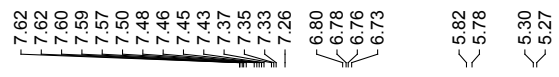

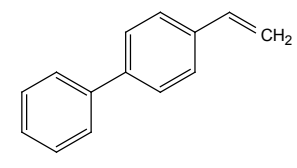

$2 u$

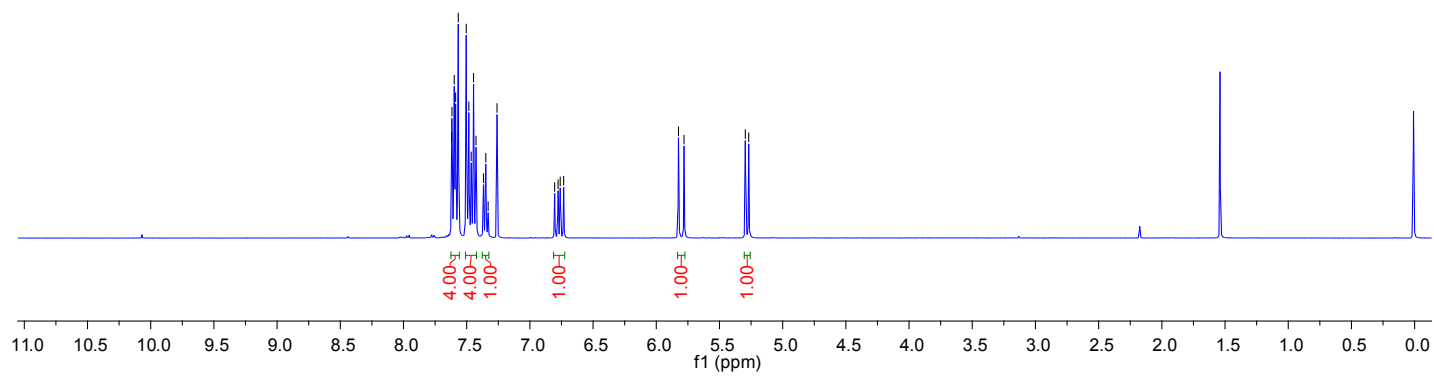

Figure S42. ${ }^{13} \mathrm{C}$ NMR spectrum $\left(100 \mathrm{MHz} \mathrm{CDCl}_{3}\right)$ of compound $2 \mathrm{u}$
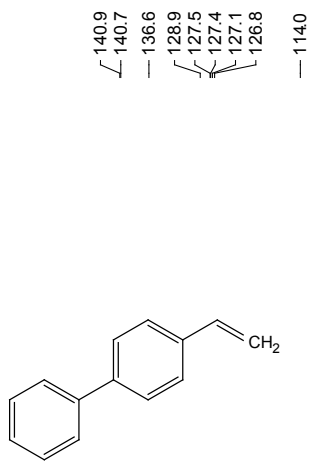

$2 u$

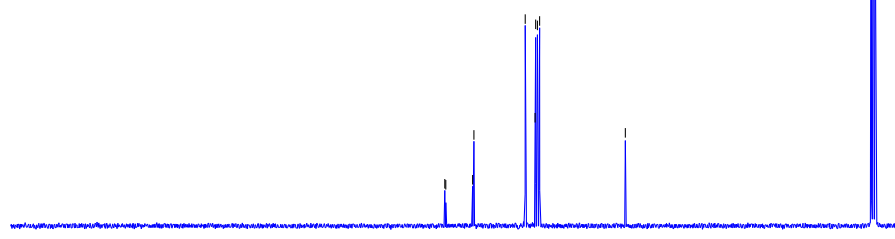

$200 \quad 190 \quad 180 \quad 170 \quad 160 \quad 150$

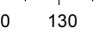

120

100
$\mathrm{f} 1(\mathrm{ppm})$ 
Figure S43. ${ }^{1} \mathrm{H}$ NMR spectrum (400 $\mathrm{MHz}, \mathrm{CDCl}_{3}$, TMS) of compound 2v

\}

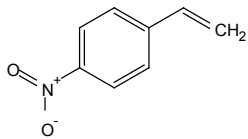

$2 v$

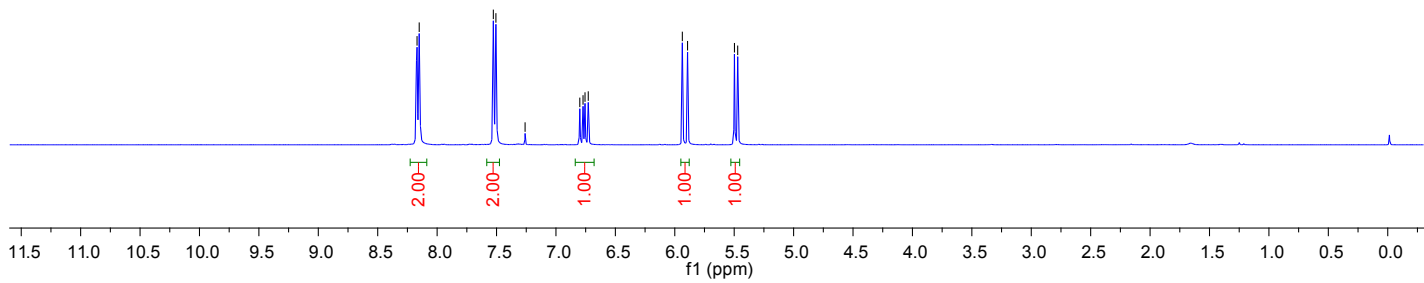

Figure S44. ${ }^{13} \mathrm{C}$ NMR spectrum $\left(100 \mathrm{MHz} \mathrm{CDCl}_{3}\right)$ of compound $2 \mathrm{v}$
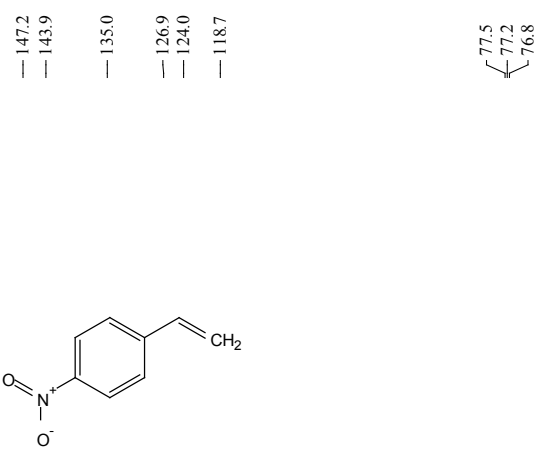

2v

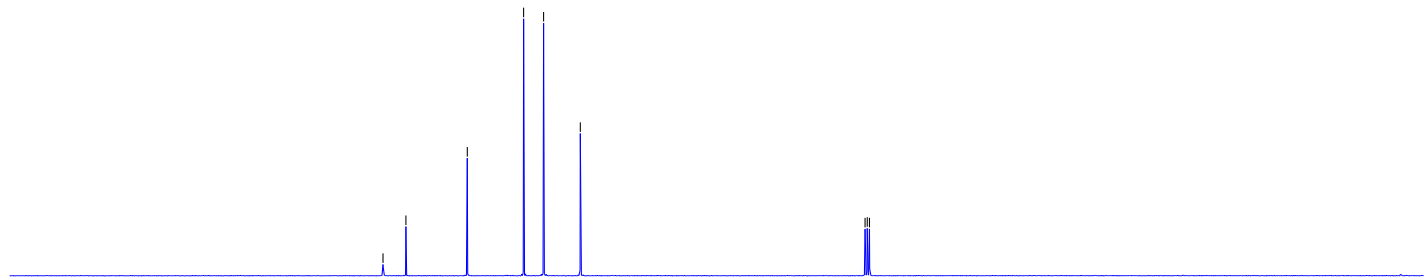

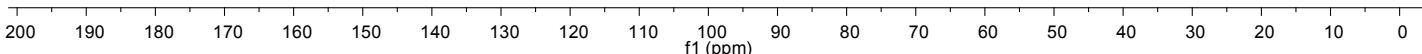


Figure S45. ${ }^{1} \mathrm{H}$ NMR spectrum (400 $\mathrm{MHz}, \mathrm{CDCl}_{3}$, TMS) of compound $2 \mathrm{w}$

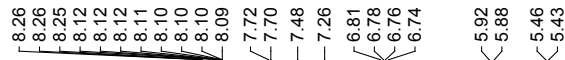

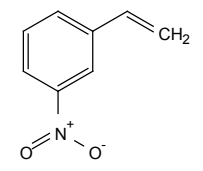

2w

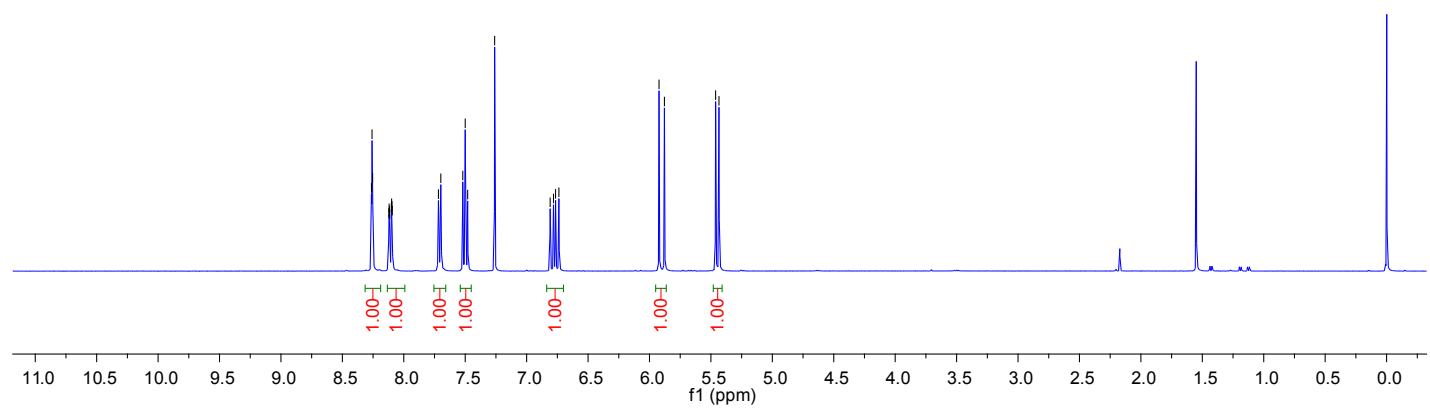

Figure S46. ${ }^{13} \mathrm{C}$ NMR spectrum $\left(100 \mathrm{MHz}, \mathrm{CDCl}_{3}\right)$ of compound 2w

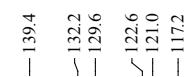

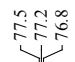

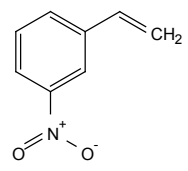

2w
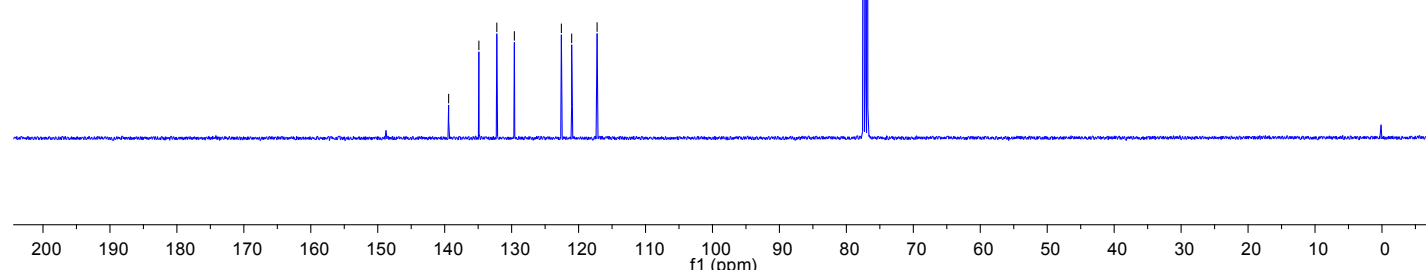
Figure S47. ${ }^{1} \mathrm{H}$ NMR spectrum (400 $\mathrm{MHz}, \mathrm{CDCl}_{3}$, TMS) of compound 2x

\}

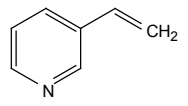

$2 x$

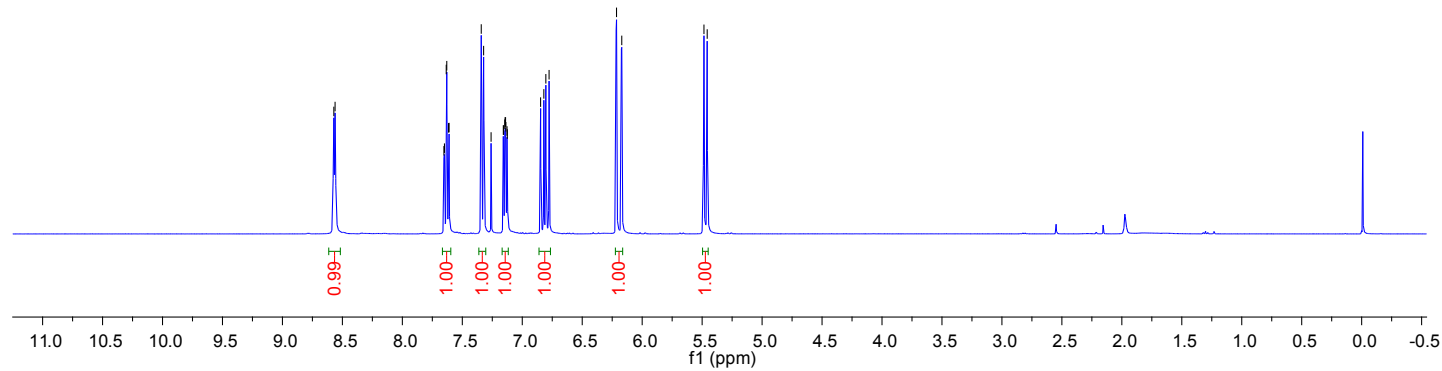

Figure S48. ${ }^{13} \mathrm{C}$ NMR spectrum (100 $\left.\mathrm{MHz}^{\mathrm{CDCl}}{ }_{3}\right)$ of compound $2 \mathrm{x}$
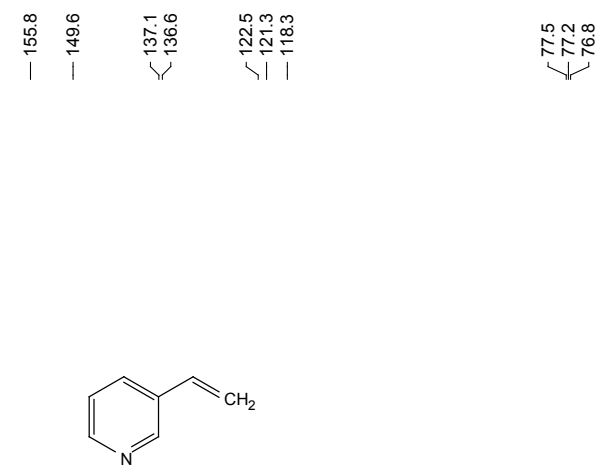

$2 x$

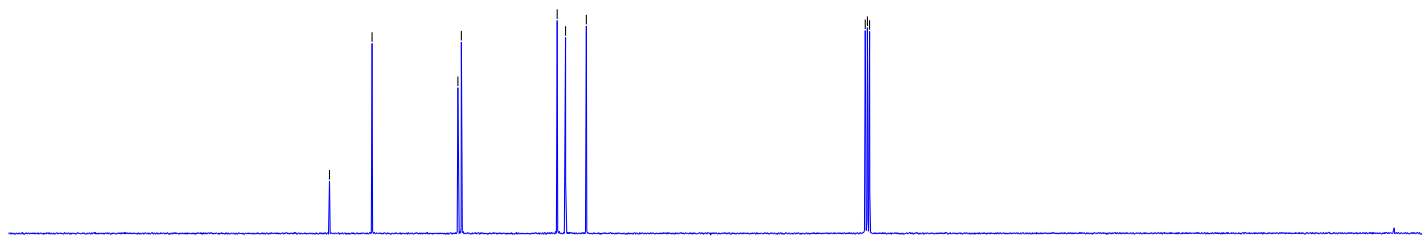

$\begin{array}{llllllllllllllllllllll}1 & 190 & 180 & 170 & 160 & 150 & 140 & 130 & 120 & 110 & \begin{array}{c}100 \\ \mathrm{f} 1(\mathrm{ppm})\end{array} & 90 & 80 & 70 & 60 & 50 & 40 & 30 & 20 & 10 & 0\end{array}$ 
Figure S49. ${ }^{1} \mathrm{H}$ NMR spectrum (400 $\mathrm{MHz}, \mathrm{CDCl}_{3}$, TMS) of compound $2 \mathrm{y}$

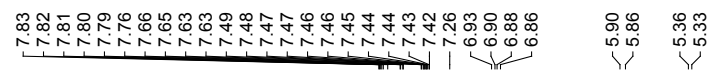

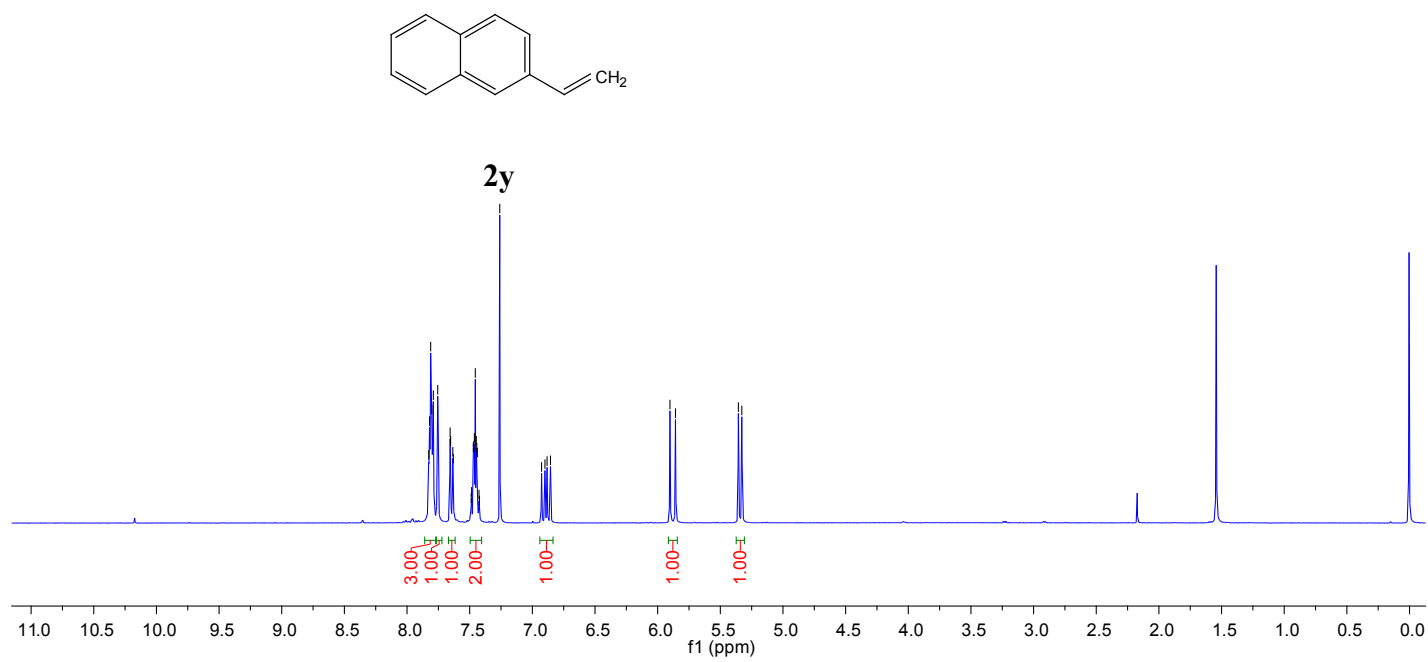

Figure S50. ${ }^{13} \mathrm{C}$ NMR spectrum (100 $\left.\mathrm{MHz}^{\mathrm{CDCl}}\right)_{3}$ of compound $2 \mathrm{y}$
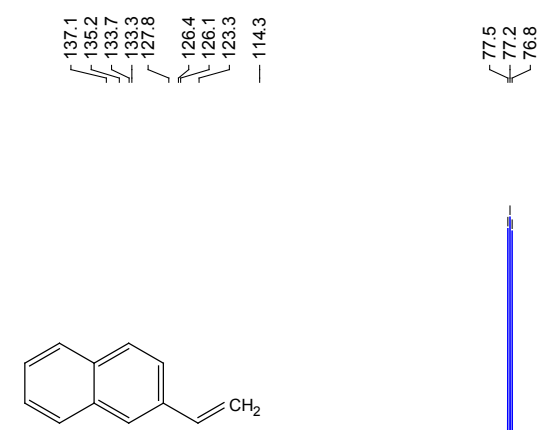

$2 y$

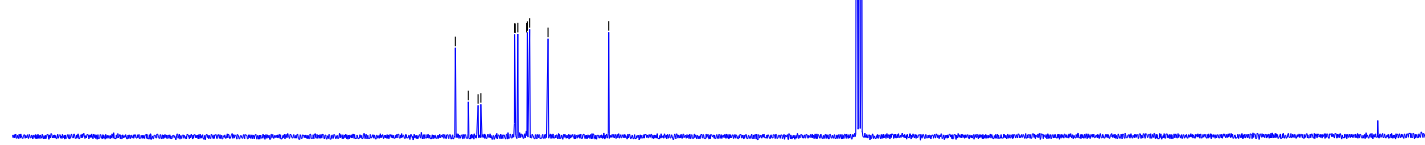

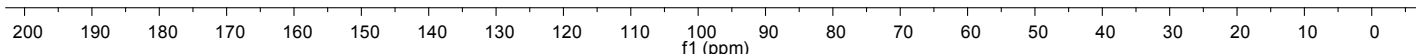


Figure S51. ${ }^{1} \mathrm{H}$ NMR spectrum (400 $\mathrm{MHz}, \mathrm{CDCl}_{3}$, TMS) of compound $2 \mathrm{z}$

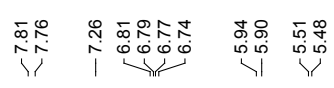
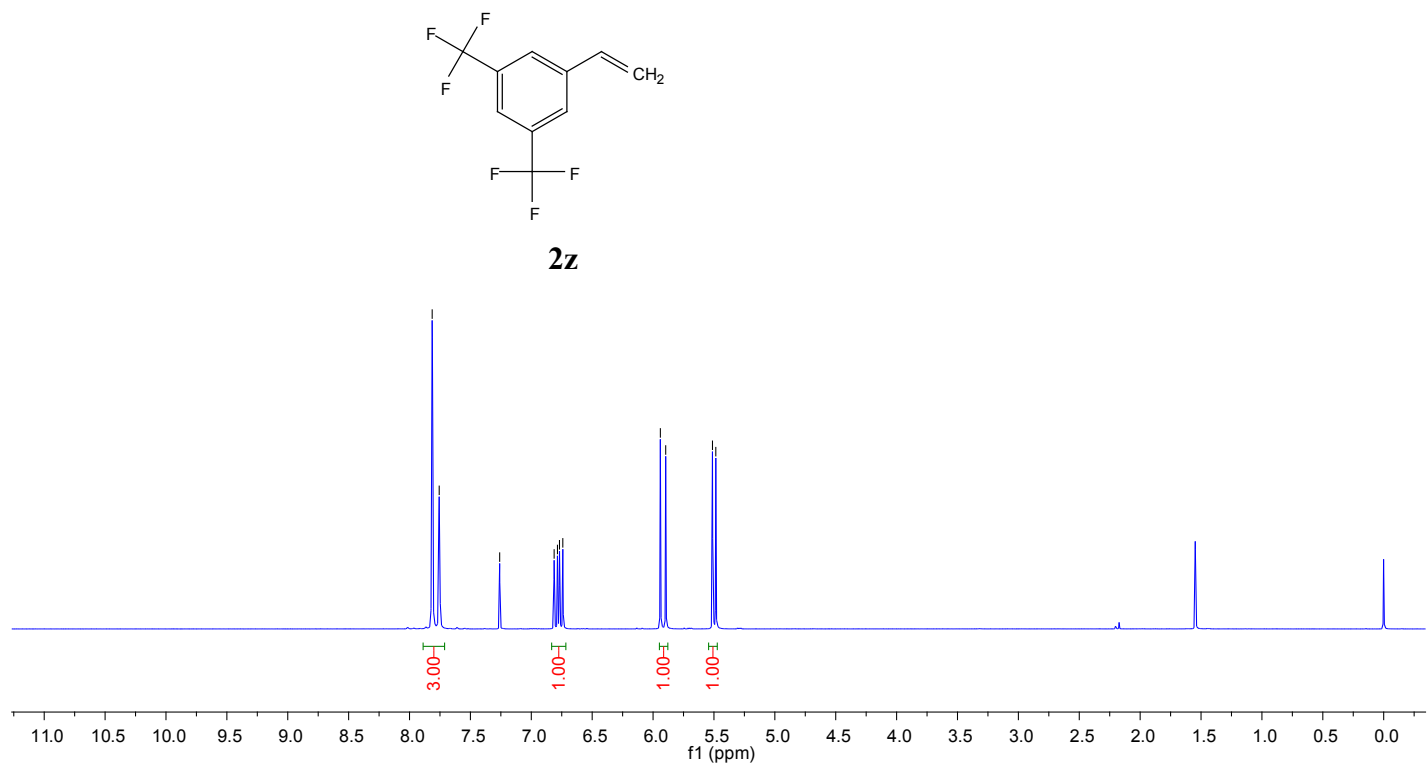

Figure S52. ${ }^{13} \mathrm{C}$ NMR spectrum $\left(100 \mathrm{MHz} \mathrm{CDCl}_{3}\right)$ of compound $2 \mathrm{z}$
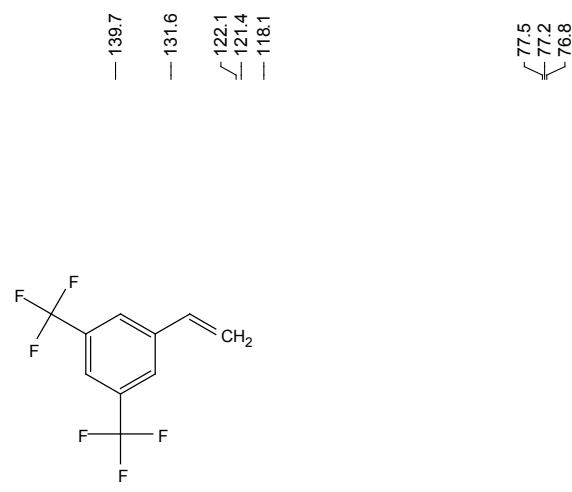

$2 z$

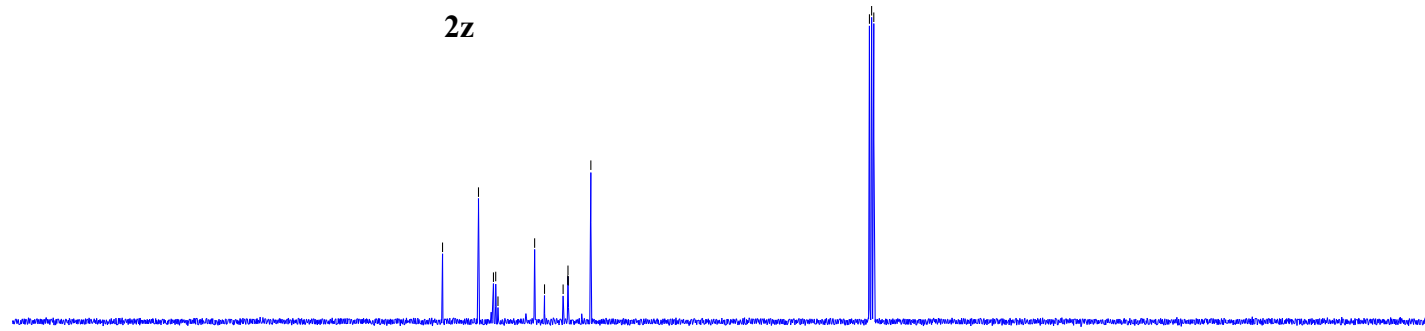

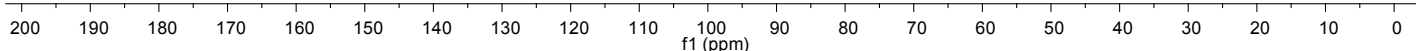


Figure S53. ${ }^{1} \mathrm{H}$ NMR spectrum (400 $\mathrm{MHz}, \mathrm{CDCl}_{3}$, TMS) of compound $2 \alpha$
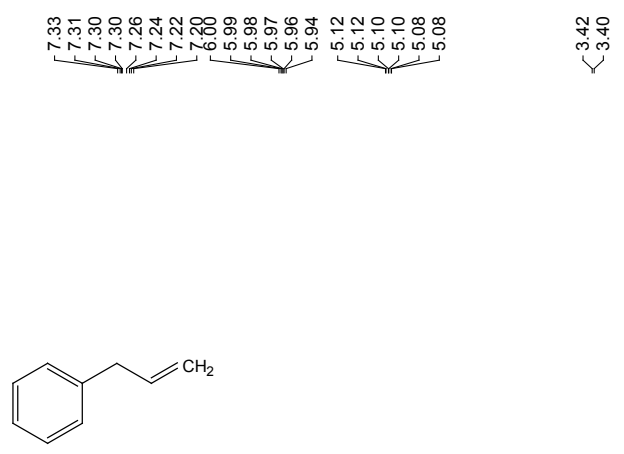

$2 \alpha$

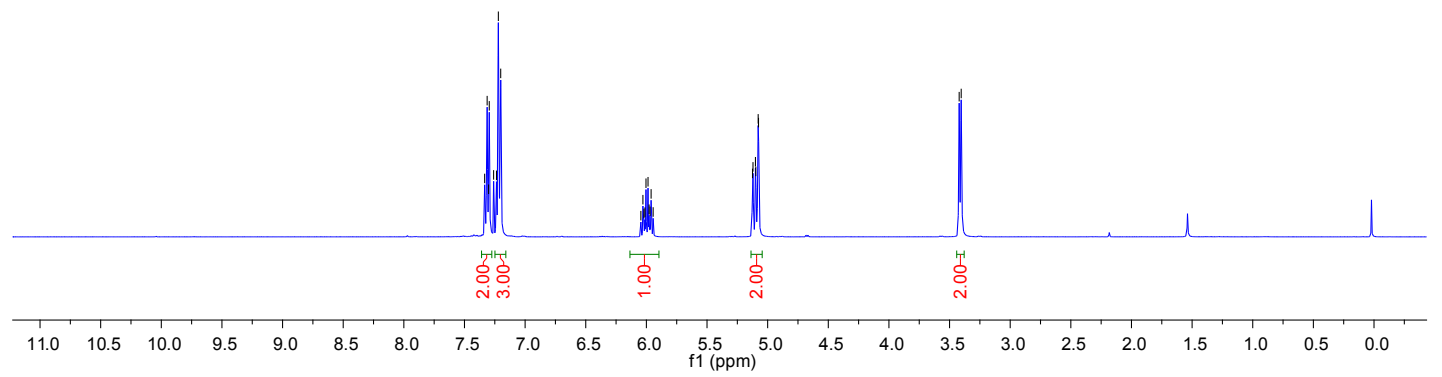

Figure S54. ${ }^{13} \mathrm{C}$ NMR spectrum $\left(100 \mathrm{MHz}, \mathrm{CDCl}_{3}\right)$ of compound $2 \alpha$
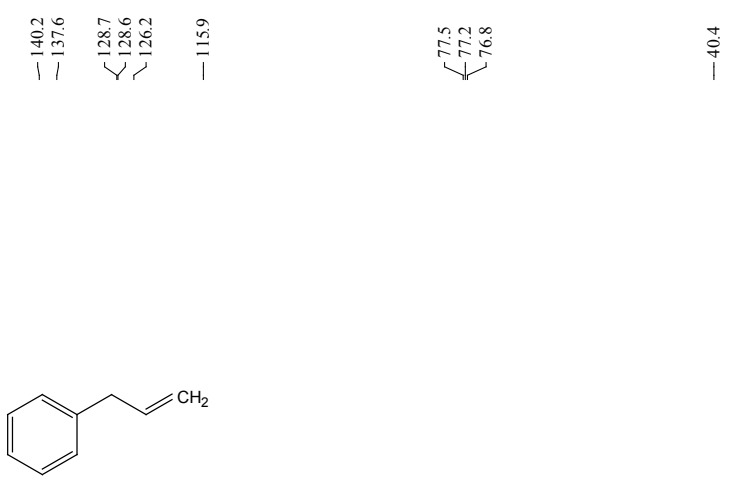

$2 \alpha$

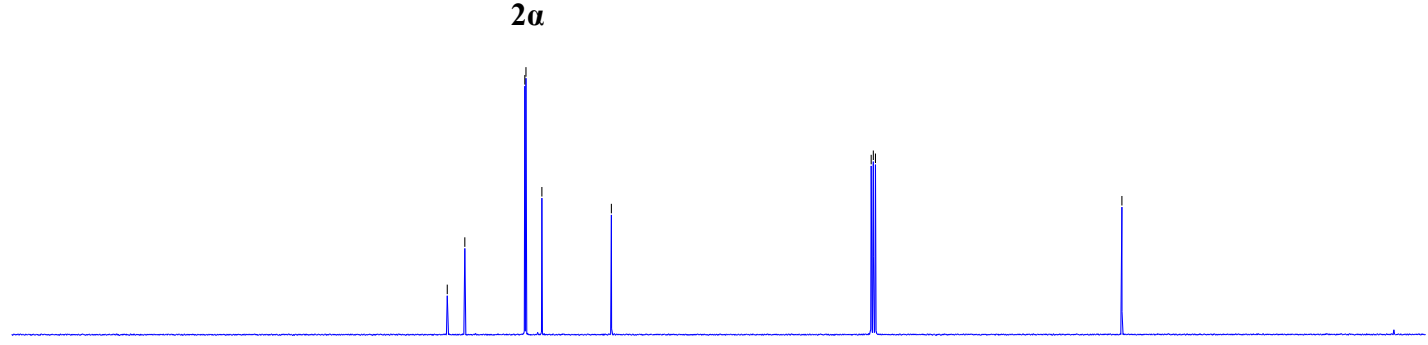

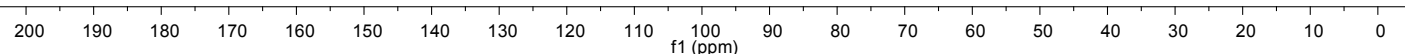


Figure S55. ${ }^{1} \mathrm{H}$ NMR spectrum (400 $\mathrm{MHz}, \mathrm{CDCl}_{3}$, TMS) of compound $2 \beta$
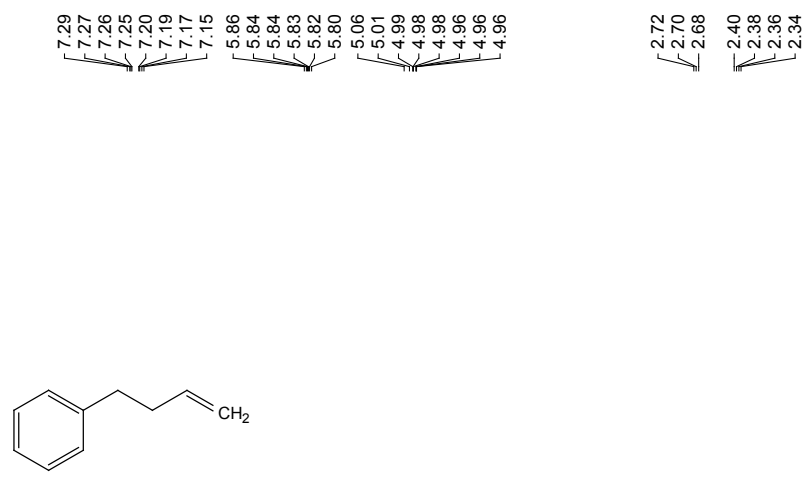

$2 \beta$

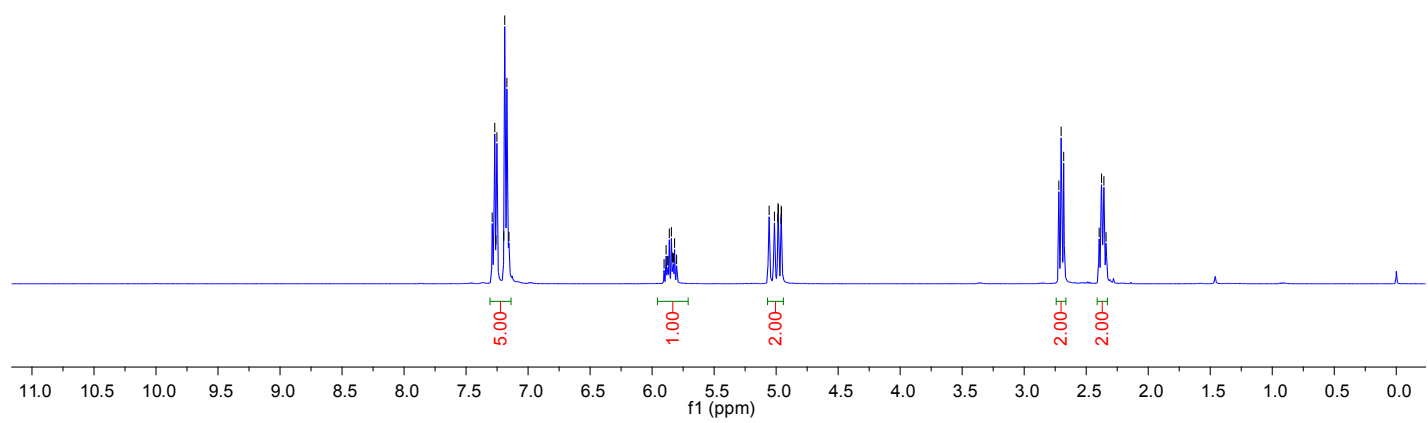

Figure S56. ${ }^{13} \mathrm{C}$ NMR spectrum (100 $\left.\mathrm{MHz}^{\mathrm{CDCl}}{ }_{3}\right)$ of compound $2 \beta$
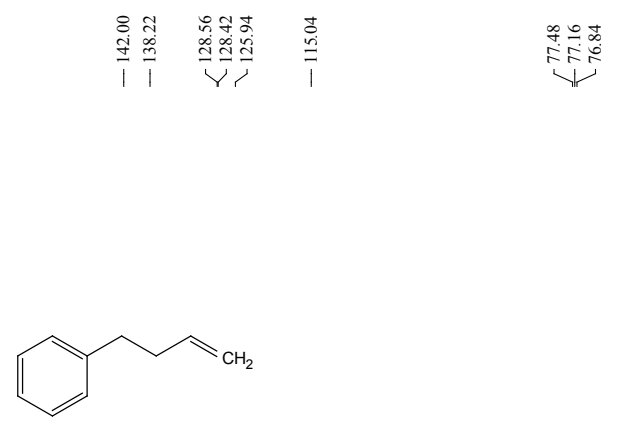

$2 \beta$

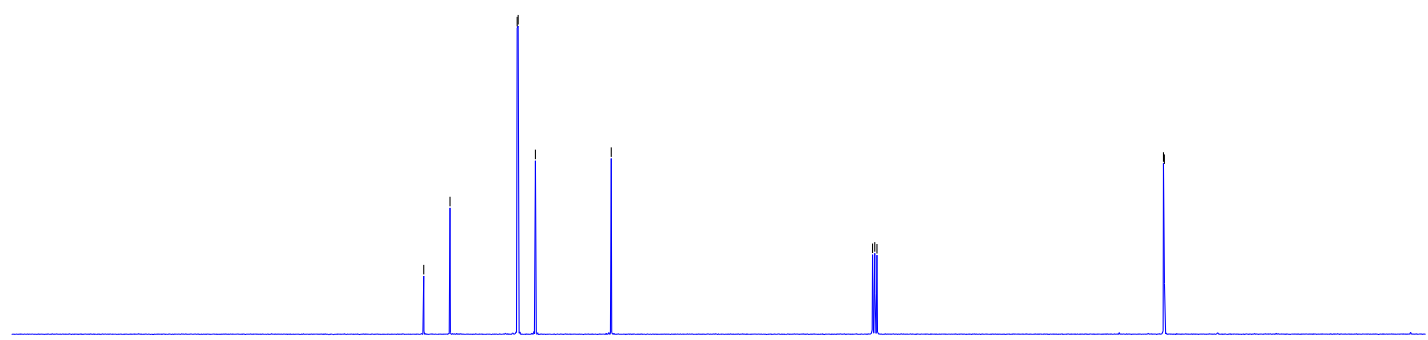

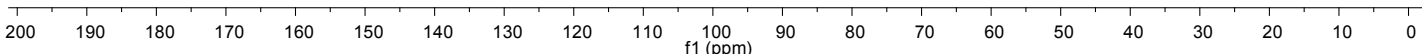


Figure S57. ${ }^{1} \mathrm{H}$ NMR spectrum (400 $\mathrm{MHz}, \mathrm{CDCl}_{3}$, TMS) of compound $2 \gamma$

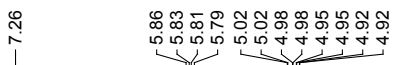

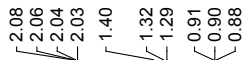

$\mathrm{N}_{\mathrm{CH}_{2}}$

$2 \gamma$

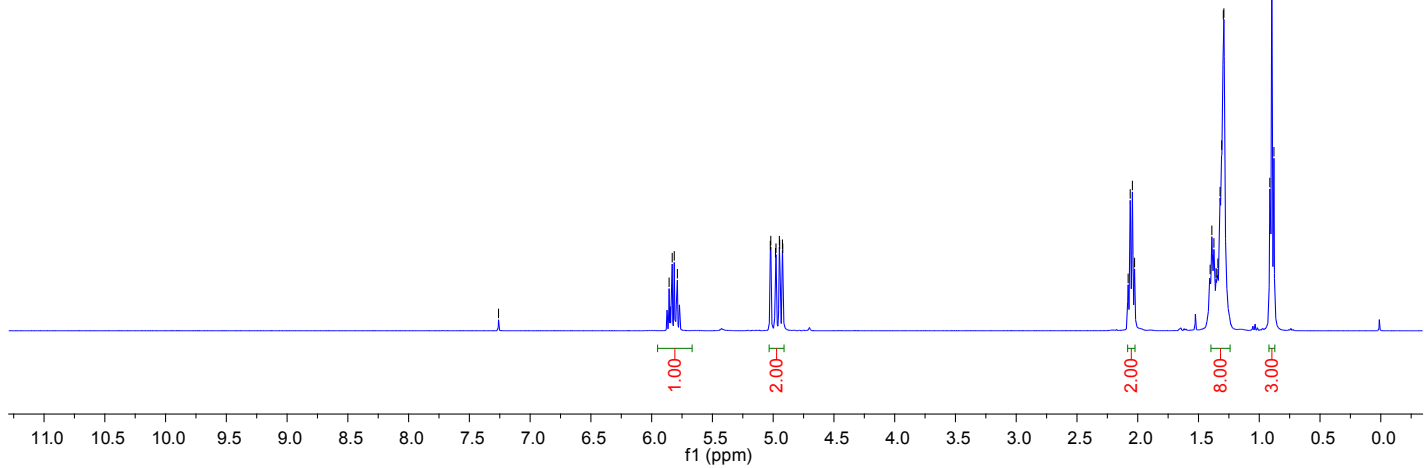

Figure S58. ${ }^{13} \mathrm{C}$ NMR spectrum $\left(100 \mathrm{MHz} \mathrm{CDCl}_{3}\right)$ of compound $2 \gamma$

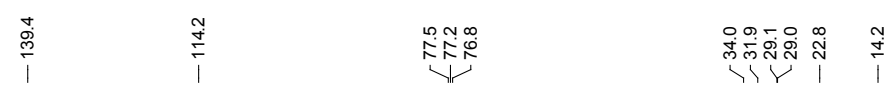

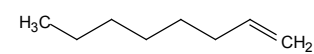

$2 \gamma$

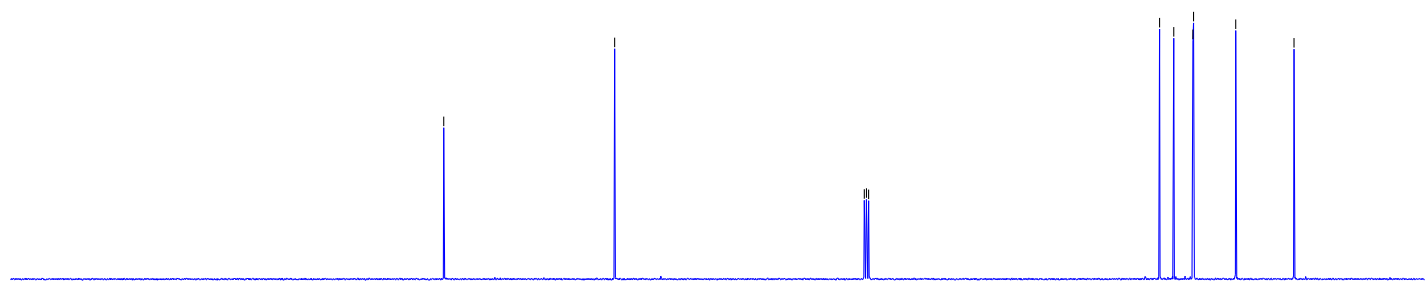

200

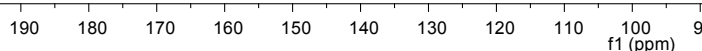

$\begin{array}{llllllll}70 & 60 & 50 & 40 & 30 & 20 & 10 & 0\end{array}$ 
Figure S59. ${ }^{1} \mathrm{H}$ NMR spectrum (400 $\mathrm{MHz}, \mathrm{CDCl}_{3}$, TMS) of compound $2 \phi$

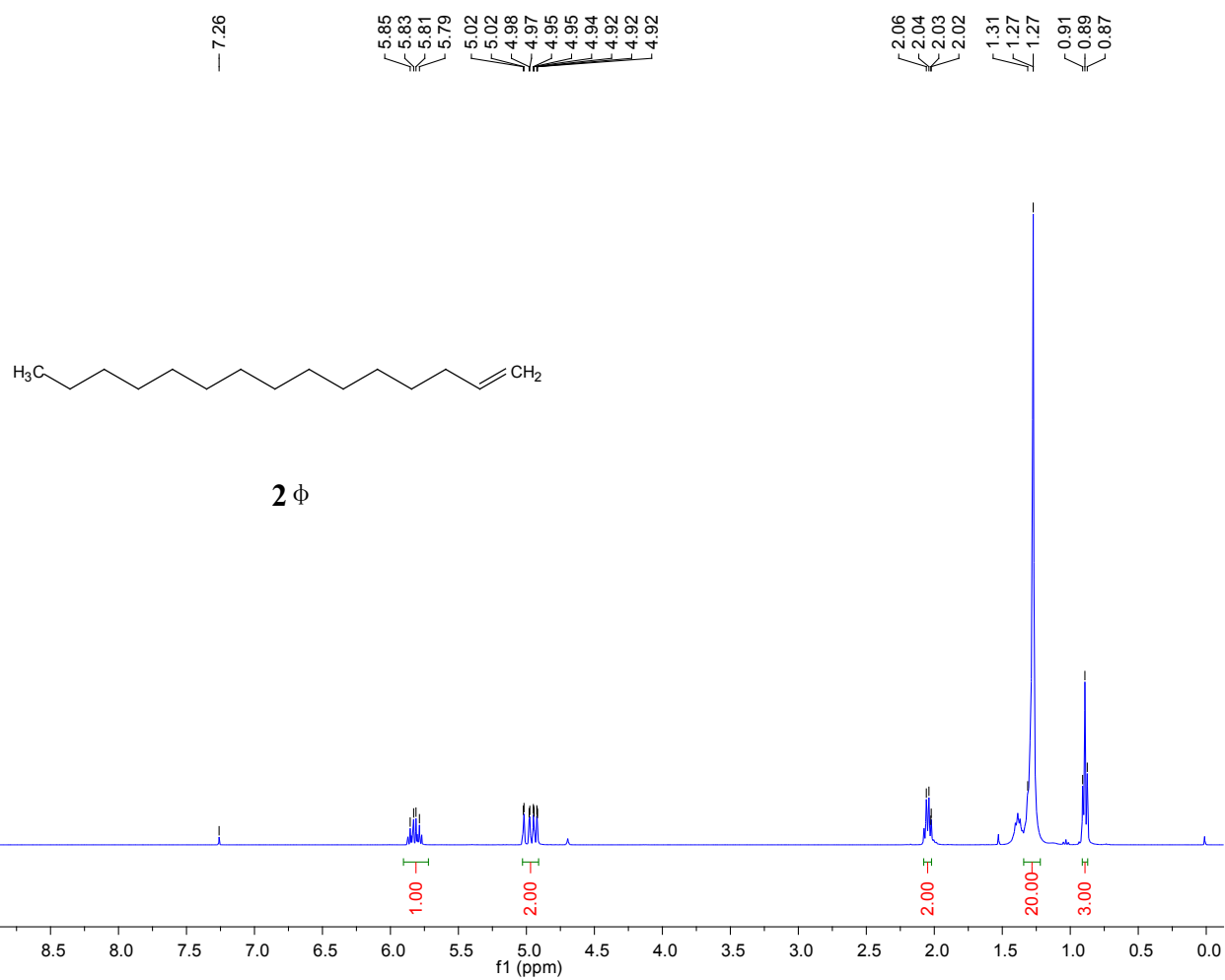

Figure S60. ${ }^{13} \mathrm{C} \mathrm{NMR}$ spectrum $\left(100 \mathrm{MHz}, \mathrm{CDCl}_{3}\right)$ of compound $2 \phi$

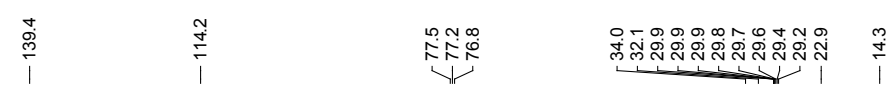

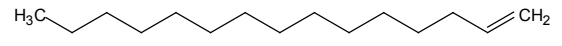

$2 \phi$

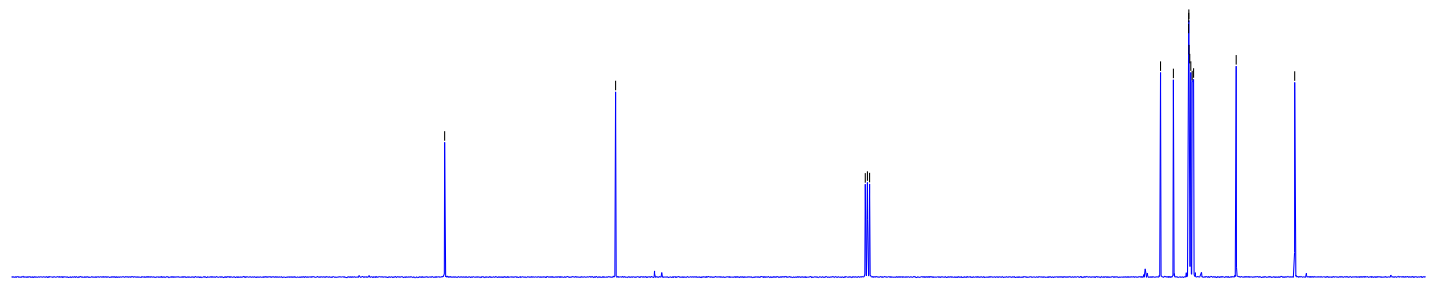

200 $\begin{array}{llllllllll}190 & 180 & 170 & 160 & 150 & 140 & 130 & 120 & 110 & 100 \\ \mathrm{f} 1(\mathrm{ppm}) & 9\end{array}$ 